\title{
NeuroImage
}

ELSEVIER

www.elsevier.com/locate/ynimg

NeuroImage 21 (2004) 1300-1319

\section{Bayesian model averaging in EEG/MEG imaging}

\author{
Nelson J. Trujillo-Barreto, ${ }^{*}$ Eduardo Aubert-Vázquez, and Pedro A. Valdés-Sosa
}

Cuban Neuroscience Center, Havana, Cuba

Received 10 June 2003; revised 3 November 2003; accepted 4 November 2003

\begin{abstract}
In this paper, the Bayesian Theory is used to formulate the Inverse Problem (IP) of the EEG/MEG. This formulation offers a comparison framework for the wide range of inverse methods available and allows us to address the problem of model uncertainty that arises when dealing with different solutions for a single data. In this case, each model is defined by the set of assumptions of the inverse method used, as well as by the functional dependence between the data and the Primary Current Density (PCD) inside the brain. The key point is that the Bayesian Theory not only provides for posterior estimates of the parameters of interest (the PCD) for a given model, but also gives the possibility of finding posterior expected utilities unconditional on the models assumed. In the present work, this is achieved by considering a third level of inference that has been systematically omitted by previous Bayesian formulations of the IP. This level is known as Bayesian model averaging (BMA). The new approach is illustrated in the case of considering different anatomical constraints for solving the IP of the EEG in the frequency domain. This methodology allows us to address two of the main problems that affect linear inverse solutions (LIS): (a) the existence of ghost sources and (b) the tendency to underestimate deep activity. Both simulated and real experimental data are used to demonstrate the capabilities of the BMA approach, and some of the results are compared with the solutions obtained using the popular lowresolution electromagnetic tomography (LORETA) and its anatomically constraint version (cLORETA).

(c) 2004 Elsevier Inc. All rights reserved.
\end{abstract}

Keywords: Bayesian model averaging; EEG; MEG; Inverse problem; Bayesian inference; Model comparison

\section{Introduction}

Our interest lies in the identification of electro/magnetoencephalogram (EEG/MEG) generators, that is, the distribution of current sources inside the brain that generate the voltage-magnetic field measured over an array of sensors distributed on the scalp surface. This is known as the Inverse Problem (IP) of the EEG/ MEG.

* Corresponding author. Cuban Neuroscience Center, Ave. 25, Esq. 158, No. 15202, P.O. Box 6412/6414, Cubanacan, Playa, Ciudad Havana, Cuba. Fax: +53-7-208-6707.

E-mail address: trujillo@cneuro.edu.cu (N.J. Trujillo-Barreto).

Available online on ScienceDirect (www.sciencedirect.com.)
Much literature has been devoted to the solution of this problem. The main difficulty stems from its ill-posed character due to the nonuniqueness of the solution, which is caused by the existence of silent sources that cannot be measured over the scalp surface. Additional complications that arise when dealing with actual data are related to the limited number of sensors available, making the problem highly underdetermined, as well as to the numerical instability of the solution, given by its high sensitivity to measurement noise.

The usual way to deal with these difficulties has been to include additional information or constraints about the physical and mathematical properties of the current sources inside the head, which limit the space of possible solutions. This has resulted in the emergence of a great variety of methods, each depending on the kind of information that has been introduced and resulting consequently in many different unique solutions.

Some methods handle the many-to-one nature of the problem by characterizing the sources in terms of a limited number of current dipoles that are fitted to the data through the minimization of some measure of the reconstruction error (De Munck, 1989; Nunez, 1981; Scherg and von Cramon, 1986; Scholz and Schwierz, 1994). These dipolar solutions have been widely used in the analysis of specific sensory and motor cortex data, where the EEG/MEG is originated by the activation of small masses of neurons (Picton et al., 1999), but have often failed in describing the spatial extension of more widespread activity, as is the case of cognitive processes and certain pathologies.

Recently, the growing experimental evidence about the existence of more diffuse brain networks has led to the emergence of the so-called distributed inverse solutions (DIS). The modeling in this sense has dramatically evolved from simple 2D approaches (Gorodnitzky et al., 1992; Hämäläinen and Ilmoniemi, 1984) to more sophisticated 3D implementations (Dale and Sereno, 1993; Fuchs et al., 1995; Gorodnistky et al., 1995; Ioannides et al., 1989; Pascual-Marqui et al., 1994; Srebro, 1996; Valdes-Sosa et al., 2000; Wang et al., 1992; Hamalainen and Ilmoniemi, 1994). These kinds of methods are designed to cope with the nonuniqueness and the numerical instability of the problem by constraining the source space to those brain regions capable of generating voltagemagnetic fields over the scalp surface (anatomic constraints) and by regularization using different regularization operators or stabilizers (Tikhonov and Arsenin, 1977). Most of these approaches lead to linear estimation procedures, which although giving quite good results when dealing with widespread activities, they fail to recover spatially concentrated sources due to their tendency to smooth out 
activations. Advances in this respect have been obtained with the development of some nonlinear approaches in the last few years (Fuchs et al., 1999; Matsuura and Okabe, 1997).

Given the wide range of methods available, it seems that again we have to face a problem of nonuniqueness related to the selection of the most appropriate methods to be used for a given data among the host of inverse solutions at hand. In other words, we have to take into account the uncertainty about selecting a single method for modeling our data. The seminal paper by Schmidt et al. (1999) raises the first alarm in this direction by pointing out the need to consider not a single "best" solution to the electromagnetic inverse problem but a whole distribution of solutions. All the subsequent inference can be carried out upon this distribution. A more detailed discussion of Schmidt's work and its differences with respect to the present approach are commented in the last section of this paper.

This model uncertainty problem has been widely treated in the Bayesian literature in the last decade, and several solutions have been proposed and applied in many other fields of scientific research (Geweke, 1994; Green, 1995; Raftery et al., 1993; Vidakovic, 1998). In the case of neuroimaging, the Bayesian formalism has been used in the formulation of some special models, not only for EEG/MEG (Baillet and Garnero, 1997; Bosch-Bayard et al., 2001; Clarke, 1991, 1994), but also for other types of neuroimaging data, like fMRI (Everitt and Bullmore, 1999; Friston, 2002; Friston et al., 2002a,b) and even for conjoint recordings of EEG/MEG and fMRI (Trujillo-Barreto et al., 2001). Unfortunately, all these approaches limit the use of the Bayesian formalism to just inferring the value of the parameters and hyperparameters involved in the model, and consequently they do not fully exploit its power to cope with hypothesis testing and model selection. This last level of inference, which is usually omitted, is precisely what gives the answer to the problem of taking into account the model uncertainty.

In the present paper, the Bayesian framework is used to formulate the inverse problem of the EEG/MEG in a way that accounts for model uncertainty. To do this, the main aspects of the traditional Inverse Problem theory are reviewed and a Bayesian formulation of the EEG Inverse Problem for the case of Minimum Norm type methods is presented. In Application, we apply this formulation to the problem of finding posterior estimates of the current density inside the brain when different anatomical constraints are assumed to describe a given data. In Results, both simulated and real physiological data are used to demonstrate the strength of the Bayesian paradigm when compared to previous approaches. Finally, a discussion of the results and of several issues that still remain open is carried out in the last section of this paper.

\section{Theory}

\section{Forward problem}

Without loss of generality we will only consider the case of just EEG recordings. Modeling MEG or joint EEG/MEG recordings is completely analogous. The Forward Problem in this case, that is, the relation of the voltage measured over the scalp surface to a given current density distribution inside the head, is defined as

$\left.v\left(\vec{r}_{\mathrm{s}}, t\right)=\int K\left(\vec{r}_{\mathrm{s}}, \vec{r}_{\mathrm{g}}\right) \overrightarrow{j(} \vec{r}_{\mathrm{g}}, t\right) d^{3} \vec{r}_{\mathrm{g}}$ where $v\left(\vec{r}_{\mathrm{s}}, t\right)$ is the voltage measured over the scalp surface; the kernel $K\left(\vec{r}_{\mathrm{s}}, \vec{r}_{\mathrm{g}}\right)$ is the electric lead field, which summarizes the geometric and electric properties of the conducting media (brain, skull and scalp) and establishes the link between the source and sensor spaces; and $\vec{j}\left(\overrightarrow{r_{\mathrm{g}}}, t\right)$ represents the Primary Current Density (PCD). The indices $\mathrm{s}$ and $\mathrm{g}$ run, respectively, over the sensor and generator spaces and $t$ denotes time. In this equation, the lead field is known and easily calculated using the Reciprocity Theorem (Plonsey, 1963; Rush and Driscoll, 1969) or simply by solving the forward problem successively with dipole sources at various locations and orientations.

A common paradigm is to analyze spontaneous EEG or data coming from evoked steady-state responses where, instead of the time evolution of the signal, we will be more interested in its spectral content. Moreover, interest is usually on the current sources that generate the activity at a given frequency. A common type of analysis in this case is to transform the whole problem to the frequency domain using the Fourier transform. Under the assumption that all the EEG time series are observations from stationary stochastic processes, this transformation is equivalent to a principal component analysis (PCA), giving a description where the complex exponentials at each frequency are the principal components and are asymptotically independent by definition (Brillinger, 1975). In this case, the problem takes the form

$\left.v\left(\vec{r}_{\mathrm{s}}, \varpi\right)=\int K\left(\vec{r}_{\mathrm{s}}, \vec{r}_{\mathrm{g}}\right) \overrightarrow{j(} \overrightarrow{r_{\mathrm{g}}}, \varpi\right) d^{3} \vec{r}_{\mathrm{g}}$

Here the symbol $\varpi$ denotes frequency and will be omitted from now on because the analysis can be carried out independently for each frequency due to the aforementioned independence. Note that the voltage and the PCD in this equation are complex numbers.

\section{EEG/MEG inverse problem: regularization approach}

The problem of solving Eq. (1) or Eq. (2) with respect to the PCD for a given voltage corresponds to the solution of a Fredholm integral equation of the first kind, and it is known as the Inverse Problem of the EEG. The main difficulty when dealing with this kind of problems is its ill-posed character due to the nonuniqueness of the solution. In other words, there are an infinite number of PCD configurations that give the same voltage over the scalp surface.

In the ideal case, we would like to find solutions in the continuum by making minimum assumptions about the physical nature of the PCD. However, Eq. (2) has an analytical solution in very few special cases, where the assumed head geometry is sufficiently simple, as in the three concentric spheres head model (Riera et al., 1997a,b). In more general cases, the source space is digitized, going from the continuum to a discrete $3 \mathrm{D}$ grid of points constructed inside the head. This simplification reduces the inverse problem to the solution of a system of linear equations,

$\mathbf{v}_{N_{\mathrm{s}}}=\mathbf{K}_{N_{\mathrm{s}} \times 3 N_{\mathrm{g}}} \cdot \boldsymbol{j}_{3 N_{\mathrm{g}}}+\boldsymbol{\varepsilon}_{N_{\mathrm{s}}}$

where $N_{\mathrm{s}}$ and $N_{\mathrm{g}}$ are the total number of sensors and grid points, respectively. It should be noted that the $3 N_{\mathrm{g}}$ rows of the column vector $\boldsymbol{j}$ correspond to the three components of the PCD vector field for each point in the grid. In this equation, we have included the term $\varepsilon_{N_{\mathrm{s}}}$ which represents the additive instrumental noise that affects the signal recorded in the sensors. 
As $N_{\mathrm{s}} N_{\mathrm{g}}$, the solution of Eq. (3) is a highly underdetermined problem with an ill-conditioned system matrix $\mathbf{K}$ (discrete version of the electric lead field). This kind of problems is commonly solved by Tikhonov regularization (Tikhonov and Arsenin, 1977). That is, for a known regularization parameter $\lambda$, the solution of Eq. (3) is given by

$\hat{\boldsymbol{j}}(\lambda)=\underset{\mathbf{j}}{\operatorname{argmin}}\left\{\|\mathbf{v}-\mathbf{K} \cdot \boldsymbol{j}\|^{2}+\lambda^{2}\|\mathbf{H} \cdot \boldsymbol{j}\|^{2}\right\}$

where $\|\boldsymbol{x}\|^{2}$ is the square of the Frobenius' norm given by $\|\boldsymbol{x}\|^{2}=$ Trace $\left(\boldsymbol{x} \overline{\boldsymbol{x}}^{\mathrm{T}}\right)$, with $\overline{\boldsymbol{x}}$ denoting complex conjugate of the vector $\boldsymbol{x}$. The parameter $\lambda$ is commonly calculated by cross validation or by the L-curve method (Hansen, 1992) and represents the relative weight between the data fitting error term $\|\mathbf{v}-\mathbf{K} \boldsymbol{j}\|^{2}$ and some assumptions about the solution, given by the choice of $\mathbf{H}$ in the seminorm $\|\mathbf{H} \boldsymbol{j}\|^{2}$ (some examples of $\mathbf{H}$ are reviewed in PascualMarqui (1999)). The family of solutions defined by these choices is known as Minimum Norm methods (MN). form

The explicit expression for $\hat{j}(\lambda)$ in this case can be written in the

$\hat{\boldsymbol{j}}(\lambda)=\left(\mathbf{K}^{\mathrm{T}} \mathbf{K}+\lambda^{2} \mathbf{H}^{\mathrm{T}} \mathbf{H}\right)^{-1} \mathbf{K}^{\mathrm{T}} \mathbf{v}=\mathbf{T}(\lambda) \mathbf{v}$

or equivalently

$\hat{\boldsymbol{j}}(\lambda)=\left(\mathbf{H}^{\mathrm{T}} \mathbf{H}\right)^{-1} \mathbf{K}^{\mathrm{T}}\left(\mathbf{K}\left(\mathbf{H}^{\mathrm{T}} \mathbf{H}\right)^{-1} \mathbf{K}^{\mathrm{T}}+\lambda^{2} \mathbf{I}_{N_{\mathrm{s}}}\right)^{-1} \mathbf{v}=\mathbf{T}(\lambda) \mathbf{v}$

where $\mathbf{I}_{N_{\mathrm{s}}}$ is the identity matrix of size $N_{\mathrm{s}}$ (Dale et al., 2000; Tarantola, 1987). Note that in both equations, the solution relates linearly to the data (linear inverse solutions (LIS)), which reduces the problem to finding the generalized inverse $\mathbf{T}(\lambda)$ that transforms the data into the estimated PCD.

Alternative approaches using norms other than Frobenius' in Eq. (4) have been described, leading to nonlinear estimations of the PCD (nonlinear inverse solutions (NIS)) (Matsuura and Okabe, 1995, 1996). These kinds of solutions, although giving less blurred activations, show localization error values similar (in some cases even greater, see for example Fuchs et al. (1999)) to LIS, and its implementation is more complex and computationally time consuming. The present work focuses on linear solutions due to its convenient [tomographic quality] to [computational cost] ratio, but we want to remark that the Bayesian formulation described here can also be applied to nonlinear approaches as well.

\section{Mathematical and anatomical constraints}

As said before, different choices for the matrix $\mathbf{H}$ correspond to different assumptions about the properties of the solution obtained. There are two kinds of assumptions commonly used:

Mathematical constraints: assume that the solution of the problem belongs to a particular functional space.

Anatomical constraints: assume that some parts of the brain are more probable of generating a measurable voltage over the scalp surface than others.

A popular method known as low-resolution electromagnetic tomography (LORETA, Pascual-Marqui et al., 1994), for example, chooses $\mathbf{H}$ equal to the Laplacian operator $\mathbf{L}$, leading to solutions that are smooth in the sense of the second order derivative. In this case, it is a common approach to introduce the anatomical constraints by solving the inverse problem restricted to those points that belong to the gray matter. To distinguish this second approach from the original unconstrained LORETA solution, it will be designated as "constrained LORETA" (cLORETA).

At this stage, we have a wide range of methods at hand, each leading to a different solution of the EEG inverse problem. Thus we need to take into account the uncertainty introduced when selecting a single model to find an optimal solution for a given data. In the next section, a Bayesian formulation of the inverse problem of the EEG that provides an answer to this question is presented.

\section{EEG/MEG inverse problem: Bayesian approach}

As stated by MacKay (1992), “. . . in science, a central task is to develop and compare models to account for the data that are gathered. ..". In the case of EEG imaging, for a given data matrix $\mathbf{v}$ we typically consider several models $M_{k}(k=1, \ldots, K)$, each of which is assumed to depend on the vector of parameters $\boldsymbol{j}$ of interest. A Bayesian model is then defined by the functional dependence of $\mathbf{v}$ on $\boldsymbol{j}$ (see Eq. (3)) and by two probability distributions: a prior distribution $p\left(j \mid \beta, M_{k}\right)$ that gives information about the allowable values that $\boldsymbol{j}$ might take for a given model $M_{k}$; and the likelihood $\left(p\left(\mathbf{v} \mid \boldsymbol{j}, \sigma, M_{k}\right)\right.$, which states the predictions that the model $M_{k}$ makes about the data $\mathbf{v}$ when the parameter vector has a particular value $\boldsymbol{j}$. Here $\beta$ and $\sigma$ are called hyperparameters and express the degree of uncertainty about the prior assumptions and the predictions, respectively.

To deal with the task stated by MacKay, the Bayesian framework involves three different levels of inference:

Level 1: infers the parameters $\boldsymbol{j}$ for a given model $M_{k}$ and given values of $\alpha$ and $\beta$ by maximizing the posterior density $\left(p\left(j \mid \mathbf{v}, \sigma, \beta, M_{k}\right)\right.$.

Level 2: infers values for $\sigma$ and $\beta$ that maximize the posterior density $p\left(\sigma, \beta \mid \mathbf{v}, M_{k}\right)$ for a given model $M_{k}$.

Level 3: Bayesian model averaging (BMA) which addresses the problem of model selection by using the posterior densities $p\left(M_{k} \mid \mathbf{v}\right)$ of the models to carry out inference upon the parameters $\boldsymbol{j}$ without conditioning on any particular model.

There is not much difference in the outcomes of the first two levels of inference when comparing Bayesian theory with more orthodox statistics. It is in the third level where Bayesian formalism is in a class of its own, because there is no general orthodox method for solving the problem of model selection. In the following sections, these three levels of inference are discussed in detail for the case of EEG/MEG imaging.

\section{Estimation of the PCD for a given model}

At this level, a given model is considered to be true and is then estimated by maximizing the posterior distribution $p\left(j \mid \mathbf{v}, \sigma, \beta, M_{k}\right)$ for known values of the hyperparameters $\sigma$ and $\beta$ (MAP estimator) with the additional constraint that Eq. (3) holds. The Bayes rule gives an expression for this posterior distribution:

$p\left(\boldsymbol{j} \mid \mathbf{v}, \sigma, \beta, M_{k}\right)=\frac{p\left(\mathbf{v} \mid \boldsymbol{j}, \sigma, M_{k}\right) p\left(\boldsymbol{j} \mid \beta, M_{k}\right)}{p\left(\mathbf{v} \mid \sigma, \beta, M_{k}\right)}$ 
The normalizing constant $p\left(\mathbf{v} \mid \sigma, \beta, M_{k}\right)$ is called the evidence for $\sigma$ and $\beta$ and is commonly ignored because it is irrelevant at this level of inference.

The likelihood $p\left(\mathbf{v} \mid \boldsymbol{j}, \sigma, M_{k}\right)$ is defined by making assumptions about the statistical properties of the experimental noise $\varepsilon$ in Eq. (3). A reasonable assumption is that the noise in the sensors obeys a complex multinormal density with zero mean and covariance matrix $\boldsymbol{\Sigma}_{\mathbf{v}}=\frac{1}{\sigma} \mathbf{I}_{N_{\mathrm{s}}}$, where $\frac{1}{\sqrt{\sigma}}$ is the standard deviation. Under this assumption, the likelihood can be written as

$p\left(\mathbf{v} \mid \boldsymbol{j}, \sigma, M_{k}\right)=N_{N_{\mathrm{s}}}^{\mathrm{c}}\left(\mathbf{K} \cdot \boldsymbol{j}, \Sigma_{\mathbf{v}}\right)$

with $N^{\mathrm{c}}$ denoting the complex multinormal distribution (see notation in Appendix A.1).

On the other hand, defining the prior probability $p\left(\boldsymbol{j} \mid \beta, M_{k}\right)$ entails assuming some distribution over the parameters of interest, which summarizes the prior knowledge we have about them. In this case, a complex multinormal density with zero mean and covariance matrix $\Sigma_{\mathbf{j}}=\frac{1}{\beta}\left(\mathbf{H}_{k}^{\mathrm{T}} \mathbf{H}_{k}\right)^{-1}$ is assumed, where $\mathbf{H}_{k}$ denotes the choice of the mathematical and anatomical constraints for each model. That is,

$p\left(\boldsymbol{j} \mid \beta, M_{k}\right)=N_{3 N_{\mathrm{g}}}^{\mathrm{c}}\left(\mathbf{0}, \Sigma_{\mathbf{j}}\right)$

Note that all the prior information is included through the specification of the covariance structure for PCD. Substituting Eqs. (8) and (9) in Eq. (7), the posterior density takes the form

$p\left(\boldsymbol{j} \mid \mathbf{v}, \sigma, \beta, M_{k}\right) \propto N_{3 N_{\mathrm{g}}}^{\mathrm{c}}\left(E[\boldsymbol{j} \mid \mathbf{v}, \sigma, \beta, M], \operatorname{Var}\left[\boldsymbol{j} \mid \mathbf{v}, \sigma, \beta, M_{k}\right]\right)$

with posterior mean

$E\left(\boldsymbol{j} \mid \mathbf{v}, \sigma, \beta, M_{k}\right)=\sigma \mathbf{A}^{-1} \mathbf{K}^{\mathrm{T}} \mathbf{V}=\hat{\boldsymbol{j}}_{k}$

and covariance

$\operatorname{Var}\left[\boldsymbol{j} \mid \mathbf{v}, \sigma, \beta, M_{k}\right]=\mathbf{A}^{-1}$

where $\mathbf{A}=\sigma \mathbf{K}^{\mathrm{T}} \mathbf{K}+\beta \mathbf{H}_{k}^{\mathrm{T}} \mathbf{H}_{k}$ (see Appendix A.1). As we see, the expected value defined in Eq. (11) is equivalent to Eq. (5) for the PCD computed by regularization if we define $\lambda^{2}=\beta / \sigma$.

\section{Inference of the hyperparameters $\sigma$ and $\beta$}

Thus far, we have assumed that $\sigma$ and $\beta$ are known. To assign values to these hyperparameters, the posterior density $p\left(\sigma, \beta \mid \mathbf{v}, M_{k}\right)$ is maximized. Again from Bayes' rule, we have

$p\left(\sigma, \beta \mid \mathbf{v}, M_{k}\right)=\frac{p\left(\mathbf{v} \mid \sigma, \beta, M_{k}\right) p\left(\sigma, \beta \mid M_{k}\right)}{p\left(\mathbf{v} \mid M_{k}\right)}$

where $p\left(\mathbf{v} \mid M_{k}\right)$ is called the evidence for the model $M_{k}$ and will be omitted in this inference level because it is not a function of $\sigma$ and $\beta$. The data-dependent term $p\left(\mathbf{v} \mid \sigma, \beta, M_{k}\right)$ has already appeared as the normalizing constant in Eq. (7), and consequently it is calculated by integrating the numerator of that equation over $\boldsymbol{j}$, yielding (see Appendix A.1)

$p\left(\mathbf{v} \mid \sigma, \beta, M_{k}\right)=\left(\frac{\sigma}{\pi}\right)^{N_{\mathrm{s}}} \beta^{3 N_{\mathrm{g}}}|\mathbf{A}|^{-1} e^{-\sigma\|\mathbf{v}\|^{2}+\left\|\mathbf{A}^{1 / 2} \hat{\boldsymbol{j}}_{k}\right\|^{2}}$

where $|\mathbf{X}|$ denotes the determinant of matrix $\mathbf{X}$. It should be noted that in our case, this integral can be calculated analytically because both the likelihood and the prior probabilities are Gaussian densities, giving a posterior distribution that is also Gaussian.
For more complicated probability distributions, this integral is not so easy to compute and the methodology is more difficult to apply. Fortunately, quite often, the posterior probability can be locally approximated as a Gaussian around the most probable value of the parameters, and the theory presented here still holds.

A more difficult task is to define the prior $p\left(\sigma \mid \beta, M_{k}\right)$ due to the lack of knowledge about the allowable values of the hyperparameters. This missing information can be expressed by assuming a flat prior over $\log \sigma$ and $\log \beta$ because both are scale parameters. With this choice of the prior, the estimation of the hyperparameters reduces to maximize the evidence for $\sigma$ and $\beta$ in Eq. (14), yielding the following conditions:

$\beta\left\|\mathbf{H}_{k} \hat{\boldsymbol{j}}_{k}\right\|^{2}=\gamma$

$\sigma\left\|\mathbf{v}-\mathbf{K} \hat{\boldsymbol{j}}_{k}\right\|^{2}=N_{\mathrm{s}}-\gamma$

where $\gamma=3 N_{\mathrm{g}}-\beta \operatorname{Trace}\left(\mathbf{A}^{-1} \mathbf{H}_{k}^{\mathrm{T}} \mathbf{H}_{k}\right)$ and measures the effective number of parameters that are well determined by the data. Note that this way of calculating the hyperparameters differs from other approaches, based for example on misfit criteria, the use of test data and cross-validation. Gull (1989) has demonstrated why the popular use of misfit criteria is incorrect and the use of test data may be an unreliable technique unless large quantities of data are available. In the case of cross-validation, it chooses hyperparameters by comparing the prediction error on a test set that was not used to estimate the values of the parameters. In this sense, the test error is a measure of performance only of the single most probable parameter vector. The evidence, however, is a measure of plausibility of the entire posterior ensemble around the best-fit estimator. A more detailed discussion about the differences between the Bayesian framework and other popular criteria for choosing hyperparameters is carried out in the paper by MacKay (1992).

The system of Eqs. (11) and (15) defines then an iterative algorithm in which, given initial values of $\hat{j}_{k}, \sigma$ and $\beta$, optimal estimators for those quantities conditional on model $M_{k}$ are obtained.

\section{Bayesian model averaging}

As we have seen, the first two levels of inference do not differ much from the traditional view of the inverse problem. In both cases, a single model is considered to be true and the PCD inside the brain is estimated under that assumption. Nevertheless, very often we have several models at hand and we might want to infer which of those models are more plausible given the data (model selection problem).

Much of the literature on statistical analysis in this situation has focused on choosing the model $M_{k}$ that maximizes the posterior probability distribution (Smith, 1991)

$p\left(M_{k} \mid \mathbf{v}\right) \propto p\left(\mathbf{v} \mid M_{k}\right) p\left(M_{k}\right)$

This procedure may be reasonable in some specific situations, but in the general case, it is not valid because it does not take into account the uncertainty associated with selecting a single model to describe the data, leading to overconfident inferences and very risky decisions.

On the other hand, Bayesian model averaging (BMA) offers an alternative way that has been the center of attention of part of the 
Bayesian community during the last few years (a good tutorial can be seen in Hoeting et al., 1999) because it provides a coherent mechanism for accounting for model uncertainty. In the present work, we will adopt the point of view proposed by Kass and Raftery (1994), who made use of the so-called Bayes' factors to compute posterior expected utilities in a way that accounts for model uncertainty.

For two given models $M_{1}$ and $M_{0}$, the Bayes' factor is defined as (see Appendix A.2)

$B_{10}=\frac{p\left(\mathbf{v} \mid M_{1}\right)}{p\left(\mathbf{v} \mid M_{0}\right)}$

Note that both the numerator and denominator are nothing but the evidences for models $M_{1}$ and $M_{0}$, respectively. Based on this definition, Bayes' factors can be interpreted as a summary of evidence provided by the data in favor of a scientific theory as opposed to another. Actually some authors use the evidence itself as a criterion for model selection, which is motivated by the fact that it can be represented as a product of two competing factors: the best-fit likelihood and an Occam's factor. In this view, the evidence reflects a tradeoff between the simplicity of the model and its capability for data fitting. A more detailed discussion about the interpretation of the evidence is reviewed in MacKay (1992).

Thus, to completely specify the Bayes' factor, we need to compute the evidences for the models under consideration. Using the normalization condition for the posterior probability $p\left(\sigma, \beta \mid \mathbf{v}, M_{k}\right)$ in Eq. (13), the evidence for any model $M_{k}$ can be calculated by

$p\left(\mathbf{v} \mid M_{k}\right)=\int p\left(\mathbf{v} \mid \sigma, \beta, M_{k}\right) p\left(\sigma, \beta \mid M_{k}\right) \mathrm{d} \sigma \mathrm{d} \beta$

The integration in this equation is commonly a difficult task due to the complicated form of the integrand. Nevertheless, when $-\log$ $p\left(\mathbf{v} \mid j, \sigma, M_{k}\right)$ and $-\log p\left(j \mid \beta, M_{k}\right)$ are quadratic forms (which is our case), the density $-\log p\left(\mathbf{v} \mid \sigma, \beta, M_{k}\right)$ reaches a single maximum at its mode $\hat{\sigma}, \hat{\beta}$ (MacKay, 1992), and the integral can be well approximated by

$p\left(\mathbf{v} \mid M_{k}\right) \approx p\left(\mathbf{v} \mid \hat{\sigma}, \hat{\beta}, M_{k}\right) p\left(\hat{\sigma}, \hat{\beta} \mid M_{k}\right) 2 \pi \Delta \log \sigma \Delta \log \beta$

where

$$
\begin{aligned}
& (\Delta \log \sigma)^{2} \approx \frac{1}{\gamma} \\
& (\Delta \log \beta)^{2} \approx \frac{1}{N_{\mathrm{s}}-\gamma}
\end{aligned}
$$

are Gaussian error bars for $\log \sigma$ and $\log \beta$. For the prior $p\left(\hat{\sigma}, \hat{\beta} \mid M_{k}\right)$ we have already assumed a flat density over log $\sigma$ and $\log \beta$, which cancels out when we calculate the Bayes' factor for two given models.

When dealing with several models $M_{0}, M_{1}, \ldots, M_{K}$, we proceed by computing the Bayes' factor for each of the $K+1$ models with respect to $M_{0}$, yielding $B_{10}, \ldots, B_{K 0}$. Then, the posterior probability of $M_{k}$ is easily derived

$$
p\left(M_{k} \mid \mathbf{v}\right)=\frac{\alpha_{k} B_{k 0}}{\sum_{r=0}^{K} \alpha_{r} B_{r 0}} \quad k=0, \ldots, K
$$

where $\alpha_{k}=p\left(M_{k}\right) \mid p\left(M_{0}\right)$ and $B_{00}=1$. In particular, we may choose the prior odds $\alpha_{k}$ equal to 1 , expressing that we have no prior preference for any of the models. In general, other values of $\alpha_{k}$ may be chosen to include prior information about the relative plausibility of competing models.

As said before, the results of the previous two sections offer a way to obtain posterior estimates of $\boldsymbol{j}$ conditionally on model $M_{k}$. On the other hand, with Eq. (20), it is possible to make inference about $\boldsymbol{j}$ without conditioning by defining its posterior density given the observed data as

$p(\boldsymbol{j} \mid \mathbf{v})=\sum_{k=0}^{K} p\left(\boldsymbol{j} \mid \mathbf{v}, M_{k}\right) p\left(M_{k} \mid \mathbf{v}\right)$

Thus, the model uncertainty is taken into account by averaging the posterior distributions under each model considered, weighted by their posterior model probability. Note that the BMA strategy defined by this equation presents several advantages over other alternatives. In Raftery and Madigan (1997), for example, the authors show that averaging over all the models in this way provides better average predictive ability, as measured by a logarithmic scoring rule, than using any single model. Particularly, it can be easily seen that procedures based on selecting a single model to carry out inference upon it can be feasible only in cases where the posterior probability of one of the models is close to 1 .

Using the results of Raftery (1993), the posterior probability $p(\mathbf{j} \mid \mathbf{v})$ in Eq. (21) can be used to define the posterior mean and covariance of $\boldsymbol{j}$ as follows:

$$
\begin{aligned}
\mathrm{E}[\boldsymbol{j} \mid \mathbf{v}]= & \sum_{k=0}^{K} E\left[\boldsymbol{j} \mid \mathbf{v}, M_{k}\right] p\left(M_{k} \mid \mathbf{v}\right) \\
\operatorname{Var}[\boldsymbol{j} \mid \mathbf{v}]= & \sum_{k=0}^{K}\left(\operatorname{Var}\left[\boldsymbol{j} \mid \mathbf{v}, M_{k}\right]+\mathrm{E}\left[\boldsymbol{j} \mid \mathbf{v}, M_{k}\right]^{2}\right) p\left(M_{k} \mid \mathbf{v}\right) \\
& -\mathrm{E}[\boldsymbol{j} \mid \mathbf{v}]^{2}
\end{aligned}
$$

where the mean and covariance of $\boldsymbol{j}$ conditional on each model $M_{k}$ are given by Eqs. (11) and (12), respectively. Note here that we have omitted the dependence on the hyperparameters because they are fixed at the values of maximum probability. As we see from these equations, the resultant Bayesian solution is an average of the solutions estimated under each model, weighted by the posterior probability of the corresponding model. This solution will then favor models that receive more support from the data and penalize those with low posterior probability values.

\section{Occam's window}

There are several practical difficulties for using Eqs. (22) and (23) when the number of models taken into account is too large, because it would entail the repeated evaluation of expectations that are commonly difficult to compute. This is critical for high dimensional problems, for which the number of variables involved in the calculations is extremely large. In neuroimaging for example, the number of parameters to be estimated usually exceeds the tens of thousands. 
This issue has been widely treated in the literature (Draper, 1995), and the general consensus has been to construct search strategies to find the sets of models that are worth taking into account in Eq. (21). One of these strategies consists on generating a Markov chain to explore the model space and then approximate Eq. (21) by the sample version of its expectation (Madigan and York, 1992). Nevertheless, these types of methods, although showing the best predictive performance, are extremely time consuming.

In the present paper, we will use the much simpler and more economic Occam's Window procedure described in Madigan and Raftery (1994) instead. In this method, the authors claim that a model that is much less likely a posteriori than the most likely one should no longer be considered, that is, models that do not belong to the set

$A=\left\{M_{k}: \frac{\max _{l}\left\{p\left(M_{l} \mid \mathbf{v}\right)\right\}}{p\left(M_{k} \mid \mathbf{v}\right)} \leq N\right\}$,

should be excluded from Eq. (21). The constant $N$ in Eq. (24) is a number much greater than $1(N=20$ is a common choice). Additionally, appealing to Occam's razor, complex models with posterior probabilities smaller than their simpler counterparts should also be excluded. These models are defined by the set

$B=\left\{M_{k}: \exists M_{l} \in A, M_{l} \subset M_{k}, \frac{p\left(M_{l} \mid \mathbf{v}\right)}{p\left(M_{k} \mid \mathbf{v}\right)}>1\right\}$.

Taking this into account, Eq. (21) is then replaced by

$p(\boldsymbol{j} \mid \mathbf{v})=\frac{\sum_{H_{k} \in C} p\left(\boldsymbol{j} \mid \mathbf{v}, M_{k}\right) p\left(\mathbf{v} \mid M_{k}\right) p\left(M_{k}\right)}{\sum_{H_{k} \in C} p\left(\mathbf{v} \mid M_{k}\right) p\left(M_{k}\right)}$

where the set $C$ is defined by $C=A \backslash B$ and is called "Occam's Window".

The strategy to identify the models in $C$ then consists of two main principles. The first principle (Occam's Window) concerns the interpretation of the ratio of posterior probabilities $p\left(M_{1} \mid \mathbf{v}\right)$ / $p\left(M_{0} \mid \mathbf{v}\right)$. Here $M_{0}$ is a model nested within $M_{1}$. The essential idea is shown in Fig. 1. If there is evidence for $M_{0}$ then $M_{1}$ is rejected, but to reject $M_{0}$ strong evidence is required for the larger model, $M_{1}$. If the evidence is inconclusive (falling in Occam's Window) neither model is rejected. The second principle is that if $M_{0}$ is rejected then so are all of the models nested within it. The final solution obtained in this way is independent of the initial set of models considered, because any initial model that includes $C$ gives a similar result.

\section{Application}

\section{Motivation}

There are two main problems that seriously affect linear inverse solutions and have captured the interest of several authors in this field:

Ghost sources: in addition to the actual sources that generate the EEG, there are additional sources in the estimated solution that do not make much physiological sense and obscure the interpretation of the results (Lütkenhöner and Grave de Peralta, 1997; Pascual-Marqui, 1995).

Increasing bias with depth: this problem is related to the underestimation of deep sources in favor of more superficial ones, leading to solutions that tend to explain the data with the generators near the sensors.

A common feature in these two situations is the difficulty of the method to correctly identify the regions of the brain that actually contribute to the generation of the EEG. In the first case, there is an overestimation of the number of sources, while in the second some of the deep generators are omitted or wrongly reconstructed. We would like then to construct a methodology that allows us to measure the adequacy of a brain region for explaining the data and use that measure to obtain solutions that automatically penalize ghost sources and favor those that really contribute to the generation of the EEG. In this respect, the Bayesian formulation described in previous sections offers a natural solution to this task.

\section{Specification of the models}

Let us assume that we have subdivided the gray matter into a finite number of compartments, and let us consider all the possible combinations of these compartments as different anatomical constraints to solve the inverse problem of the EEG. We introduce

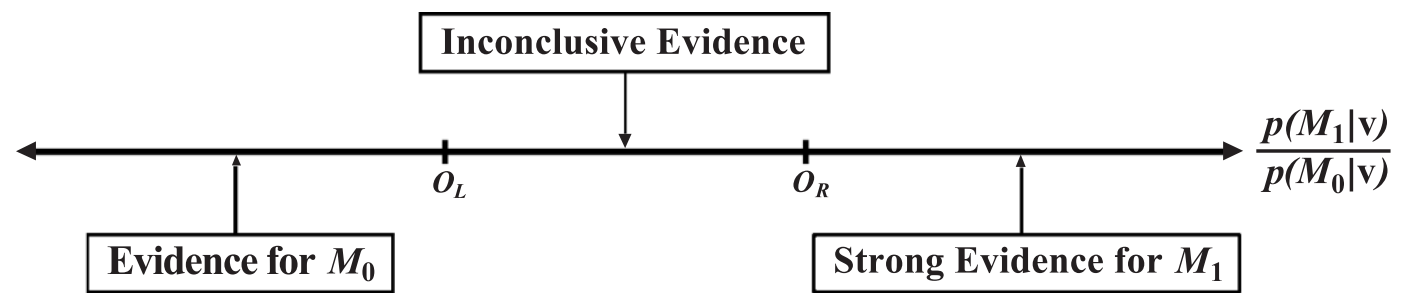

Fig. 1. Occam's Window: interpretation of the posterior probability ratio. 


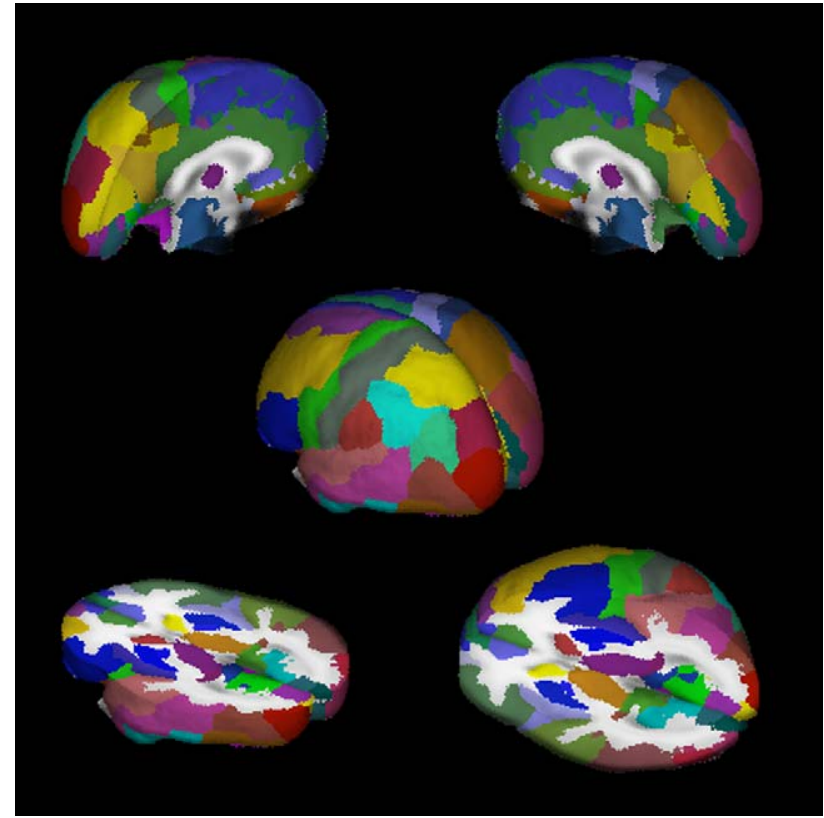

Fig. 2. 3D segmentation of 71 structures of the Probabilistic MRI Atlas developed at the Montreal Neurological Institute. As shown in the color scale, brain areas belonging to different hemispheres were segmented separately. these constraints in our formalism by defining the covariance matrix in Eq. (9) using

$\mathbf{H}_{k}=\mathbf{L}\left(\mathbf{P}_{k} \otimes \mathbf{I}_{3}\right)$

where $\mathbf{L}$ is the discrete Laplacian operator, $\mathbf{I}_{3}$ is the identity matrix of size $3, \otimes$ denotes the Kronecker product and $\mathbf{P}_{k}$ is the diagonal matrix

$\mathbf{P}_{k}=\left[\begin{array}{cccc}\frac{1}{p_{1}} & 0 & \cdots & 0 \\ 0 & \frac{1}{p_{2}} & \cdots & 0 \\ \vdots & \vdots & \ddots & \vdots \\ 0 & 0 & \cdots & \frac{1}{p_{N_{\mathrm{g}}}}\end{array}\right]$

Here $p_{i}\left(i=1, K, N_{\mathrm{g}}\right)$ are the probabilities of the grid points for belonging to the gray matter and were derived from the average Probabilistic MRI Atlas (PMA) produced by the Montreal Neurological Institute (Collins et al., 1994; Evans et al., 1993, 1994; Mazziotta et al., 1995). With this definition of $\mathbf{P}_{k}$, a particular anatomical constraint is chosen by dropping the probabilities of the points outside the region of interest to a value $\square 1$. We will call each set of $p_{i}$ 's taken in this way as probabilistic mask. Note that this parameterization of the covariance matrix reduces to the traditional cLORETA method if we take $\mathbf{P}_{k}=\mathbf{I}_{N_{\mathrm{s}}}$ (see Mathematical and anatomical constraints and Estimation of the PCD for a given model

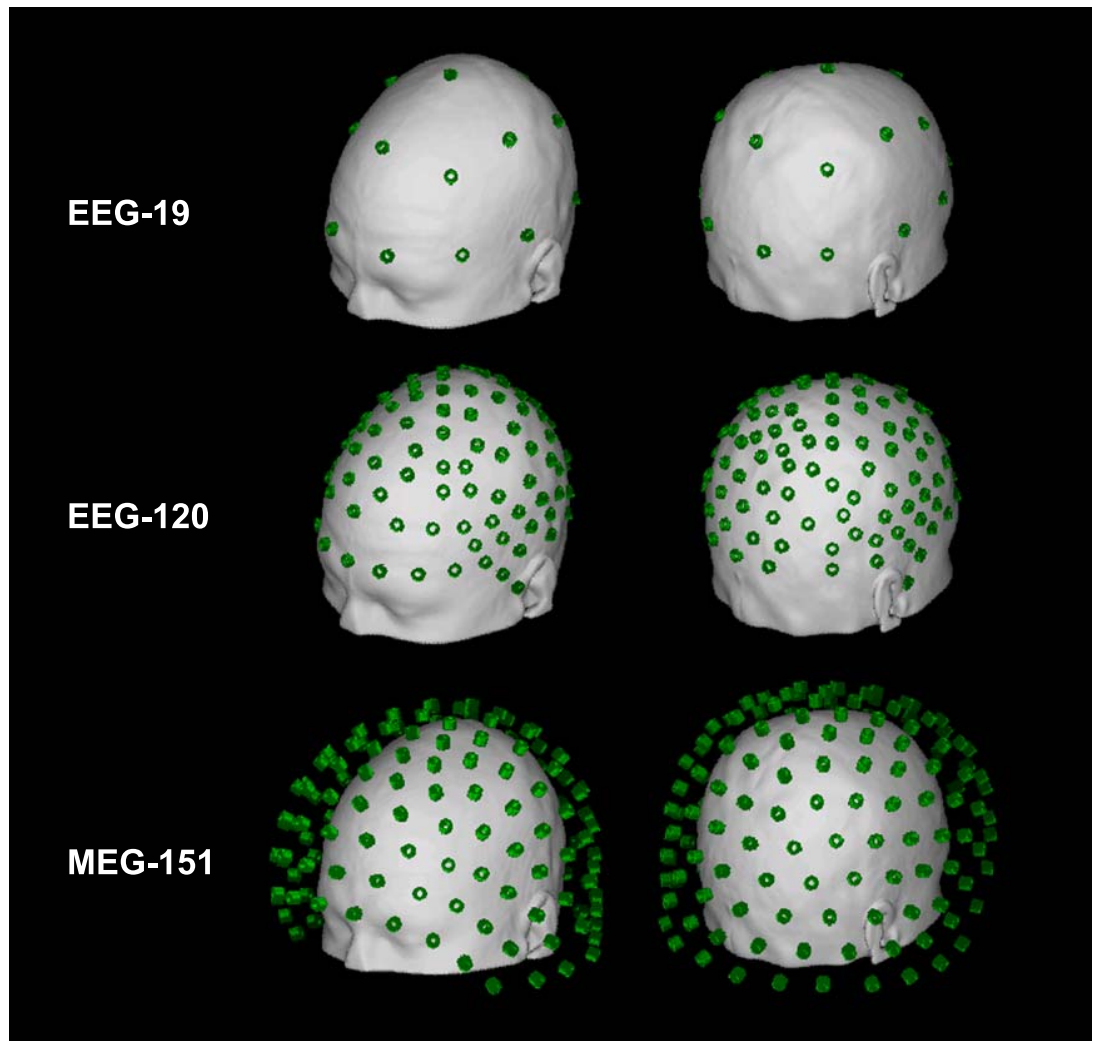

Fig. 3. Different arrays of sensors used in the simulations. EEG-19 represents the $10 / 20$ electrodes system; EEG-120 is obtained by extending and refining the 10/20 system; and MEG-151 corresponds to the spatial configuration of MEG sensors in the helmet of the CTF System Inc. 
sections), which means that the solution is calculated for all grid points within the gray matter. For the original unconstrained case (LORETA), all grid points within the head, including those that fall in the white matter, need to be taken into account. In this sense, LORETA and cLORETA could be interpreted as two of the models that are considered within the BMA paradigm.

Thus, choosing different probabilistic masks will define different prior distributions over the current density given by Eq. (9), and consequently, will represent different Bayesian models to be considered. Applying then the BMA framework to this case allows us to measure the "adequacy" of each model to a given data in terms of posterior probabilities defined in Eq (20); and to use Eq. (22) to obtain posterior estimates of the PCD inside the brain by penalizing models or regions that receive less support from the data and favoring those with higher posterior probabilities.

In the present work, we take 71 brain regions, obtained from a 3D segmentation of the PMA, as the compartments used to define the probabilistic mask corresponding to each model. Because several of these structures might be involved in a given brain process, more complex models need to be considered and were constructed by combination of the simple 71 compartments. As shown in Fig. 2, the segmentation preserves the hemispheric symmetry of the brain and includes also deep areas like thalamus, basal ganglia and brain stem.

\section{Results}

\section{Simulations}

In this section, both EEG and MEG simulated data are used to characterize the BMA approach as a tomographic method and to demonstrate its strengths in the analysis of both types of measurements. The results are also compared to previous approaches, such as traditional LORETA and cLORETA solutions.

\section{Description of the simulated data}

According to previous sections, our source space consists of a $3 \mathrm{D}$ grid of points that represent the possible generators of the EEG/ MEG inside the brain, while the measurement space is defined by the array of sensors where the EEG/MEG is recorded. In the present work, 41,850 grid points (4.25-mm grid spacing) and different arrays of electrodes or coils are placed in registration with the PMA. The 3D grid is further clipped by the gray matter,
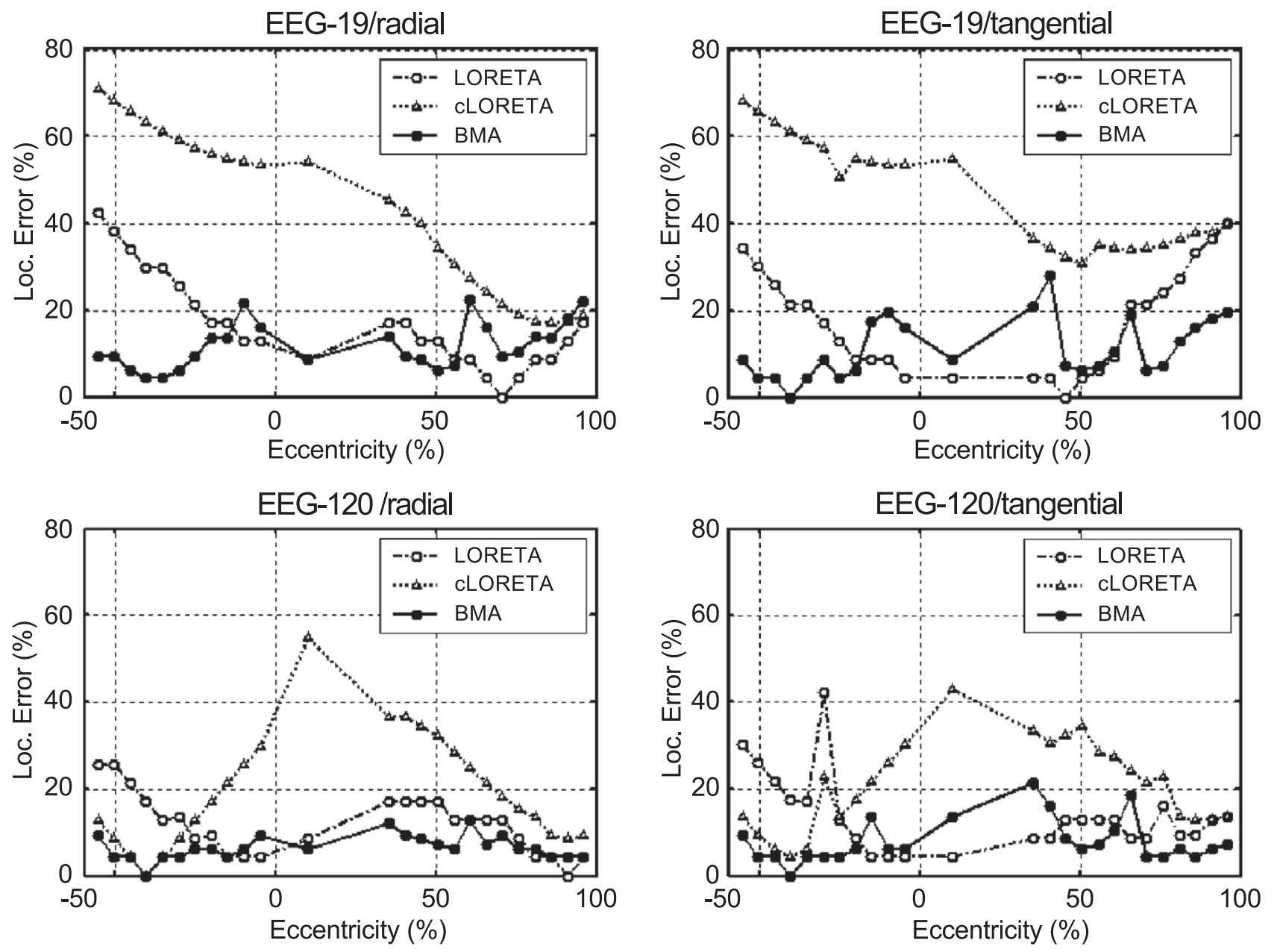

Fig. 4. Localization errors for EEG-19 and EEG-120 simulated data. The eccentricity is normalized to the head radius measured vertically along the $z$-axis $(85$ $\mathrm{mm})$ and then expressed in \%. The negative values represent test dipole positions below $z=0$. 
which consists of all brain regions segmented and shown in Fig. 2 (18,905 points). In this way, the Bayesian models (as defined in Application section) are completely specified.

The three arrays of sensors used in this study are depicted in Fig. 3. For EEG simulations, a first set of 19 electrodes (EEG-19) from the 10/20 system (FP1, FP2, F3, F4, C3, C4, P3, P4, O1, O2, F7, F8, T3, T4, T5, T6, Fz, Cz and Pz) is chosen. A second configuration of 120 electrodes (EEG-120) is also used to investigate the dependence of the results on the number of sensors. In this case, the electrodes' positions are determined by extending and refining the 10/20 system. For MEG simulations, a dense array of 151 sensors with a spatial localization that corresponds to the configuration of gradiometers in the helmet of the CTF System Inc is used (MEG-151). The physical models constructed in this way allow us to easily compute the electric and magnetic lead field matrices that relate the PCD inside the head, to the voltage and the magnetic field measured at the sensors' locations in each case.

In the present study, 25 test dipoles along the vertical axis through the center of the head are simulated. The origin of the coordinate system used here ("center of the head") is the meeting point between the axis through inion and nasion and the axis through the preauricle points of the left and right ear. The $z$-axis is then pointing upward (to the vertex of the head), while the $x$ - and $y$ - axes are in the horizontal plane pointing to the front and left side of the head, respectively. The $z$-coordinates of the test dipoles then vary from -46.75 to $80.75 \mathrm{~mm}$.

Regarding the temporal dynamics, all sources are simulated using a linear combination of sine functions with frequency components evenly spaced in the alpha band $(8-12 \mathrm{~Hz})$. The amplitude of oscillation as a function of frequencies is a narrow Gaussian peaked at $10 \mathrm{~Hz}$, with maximum of $1 \mathrm{~mA} / \mathrm{m}^{2}(1 \mathrm{nA} /$ $\mathrm{mm}^{2}$ ). That is, for each dipole position, the time course of the activity is given by

$j(t)=\sum_{i=1}^{N} A_{i} \sin \left(2 \pi f_{i} t\right)$, where $A_{i}=\mathrm{e}^{-8\left(f_{i}-10\right)^{2}} 8 \mathrm{~Hz} \leq f_{i} \leq 12 \mathrm{~Hz}$

Here, $f_{i}$ is the frequency component and $t$ denotes time. Radial (vertical) and tangential (horizontal) dipoles are used to investigate orientation-dependent effects, and the noise in each sensor was generated from a Gaussian distribution with zero mean and was added to the voltage-magnetic field calculated by solving the forward problem. To simulate realistic conditions, the noise variance was chosen to obtain a signal-to-noise ratio (SNR) of about 10 for the most superficial dipoles. Because the dipoles have the same strength time course throughout all simulations, depth-dependent
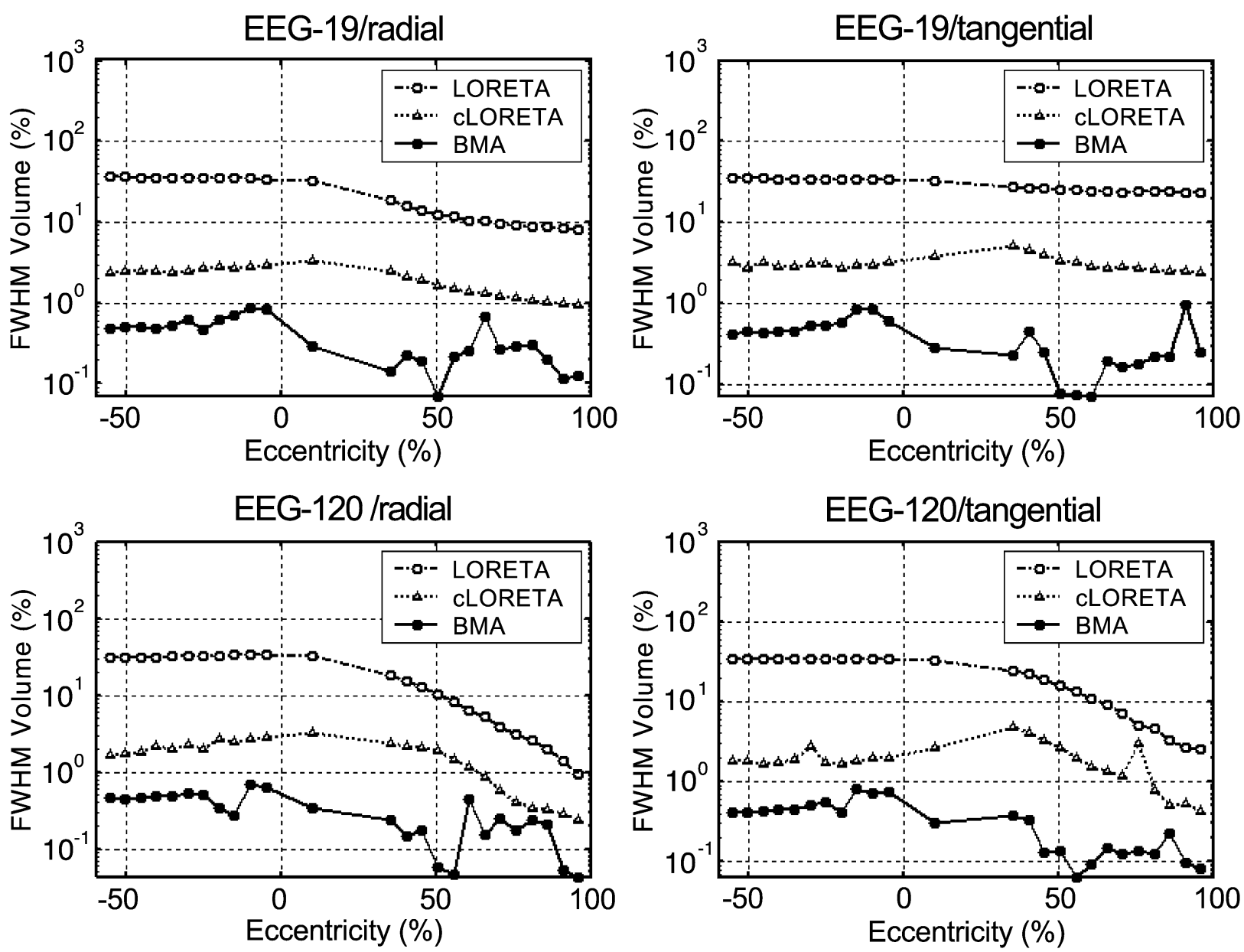

Fig. 5. Spatial resolution measured through the full with at half maximum (FWHM) volume for EEG-19- and EEG-120-simulated data. The eccentricity is normalized to the head radius measured vertically along the $z$-axis $(85 \mathrm{~mm})$ and then expressed in $\%$. The negative values represent test dipole positions below $z=0$. 
SNRs are obtained. Taking all this into account, for each test dipole, 30 artifact-free epochs (segments) of EEG/MEG, each $2 \mathrm{~s}$ long with a sample period of $5 \mathrm{~ms}$, were simulated and transformed to the frequency domain by using the Fast Fourier Transform (FFT). Finally, the BMA approach, LORETA and cLORETA are used to obtain the reconstructed current densities in each series of experiments.

\section{Localization error and spatial resolution}

To evaluate reconstruction results, the same measures evaluated by Fuchs et al. (1999) are used. In that work, a similar simulation study was carried out for comparison and characterization of different linear and nonlinear Minimum Norm approaches in terms of its mislocalization and spatial resolution. The localization error is then defined as the distance from the weighted centers of the clipped (by a $50 \%$ threshold) current distributions, to the true position $\overrightarrow{r_{0}}$ of the simulated dipole, that is

$\|\Delta \vec{r}\|=\left\|\sum_{i} j_{i} \cdot \overrightarrow{r_{i}} / \sum_{i} j_{i}-\overrightarrow{r_{o}}\right\|, \quad$ with $j_{i}>0.5 j_{\max }$

where $\vec{r}_{i}$ is the position of the $i$ th voxel with activity above the $50 \%$ of maximum activation and $j_{i}$ is the absolute value of the PCD at that voxel. The resolution of the methods is determined by the full with at half maximum (FWHM) volume, which as in Fuchs' paper is calculated by counting all voxels with strength above $50 \%$ of the maximum current and multiplying that number by the volume of the voxel $\left(4.25 \times 4.25 \times 4.25 \mathrm{~mm}^{3}=18.06 \mathrm{~mm}^{3}\right)$. The localization errors are further normalized to the head radius measured vertically along the $z$-axis $(85 \mathrm{~mm})$, and the FWHM volumes are normalized to the volume of the sphere defined by that radius. The two relative magnitudes are then expressed in percent.

\section{Reconstructions results}

The localization errors for EEG-19 and EEG-120 data and for the three approaches under analysis are depicted in Fig. 4. There is a general decrease of the localization error for all methods as the number of electrodes increases, although the major changes are undergone by LORETA and cLORETA for eccentricities below $-20 \%$. For greater eccentricities, BMA and LORETA show a similar behavior, with localization errors below 20\%. Much greater error values are shown by cLORETA, which tend to decrease for very large eccentricities. Note that, on the contrary of LORETA approaches, no trend with depth is observed for BMA, which keeps very small localization errors for all eccentricity values. No significant dipole orientation effects are observed in any of the cases.
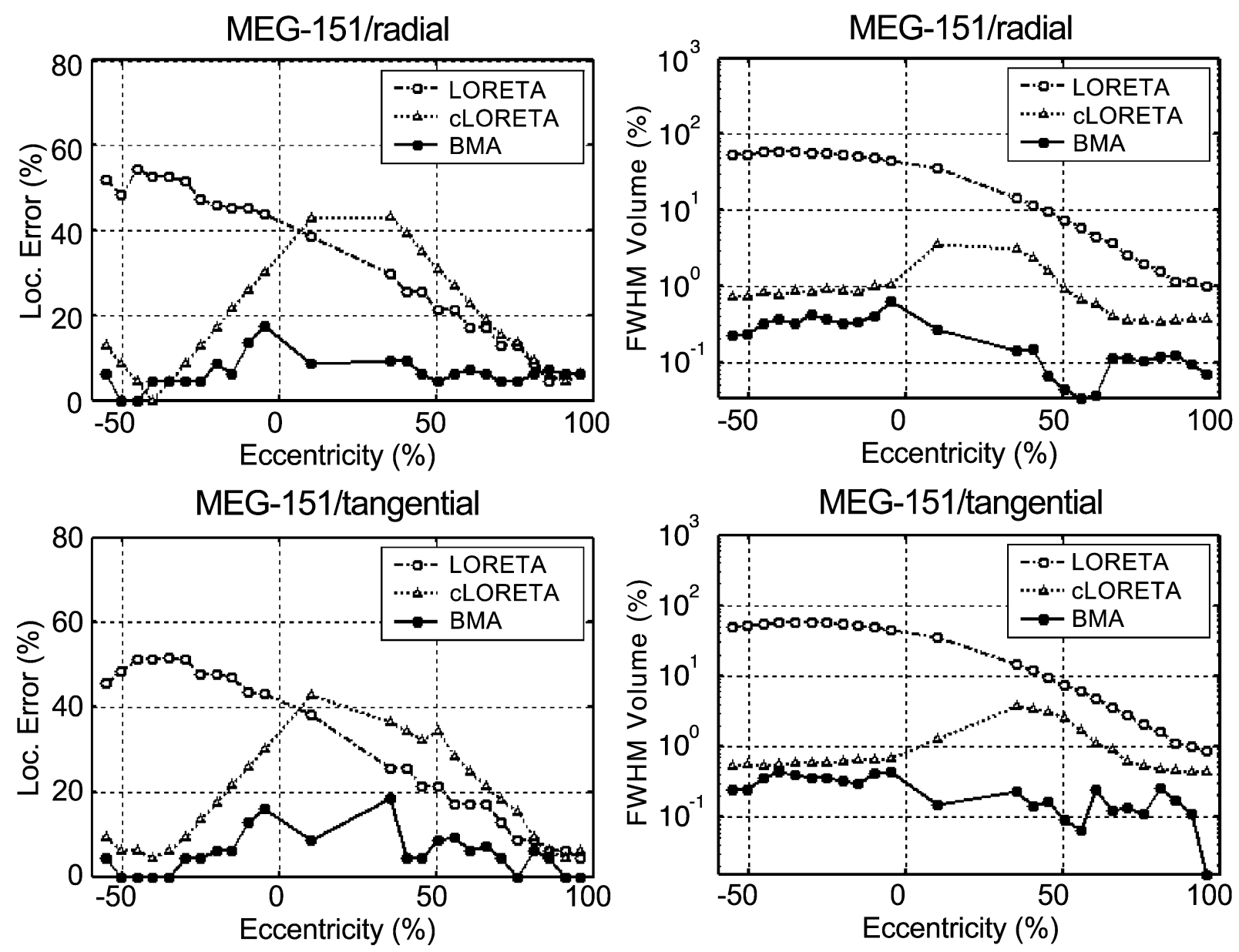

Fig. 6. Localization errors and spatial resolution measured through the full with at half maximum (FWHM) volume for MEG-151-simulated data. The eccentricity is normalized to the head radius measured vertically along the $z$-axis $(85 \mathrm{~mm})$ and then expressed in \%. The negative values represent test dipole positions below $z=0$. 


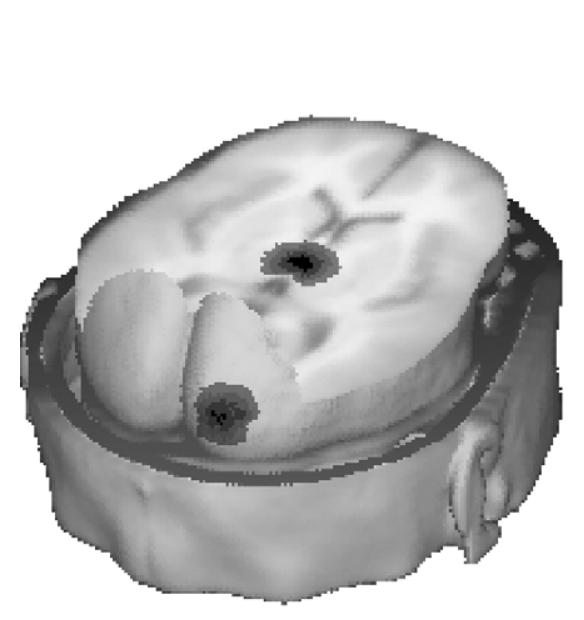

A

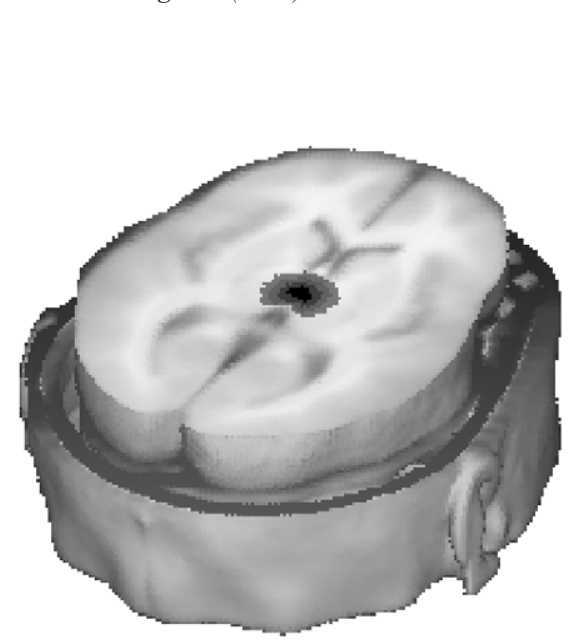

B

Fig. 7. Spatial distributions of the simulated primary current densities. (A) Simultaneous activation of two sources at different depths: one in the occipital pole right and the other in the thalamus $(\mathrm{OPr}+\mathrm{Th})$. (B) Simulation of a deep source in the thalamus (Th).

\section{CLORETA}

EEG-19

EEG-120

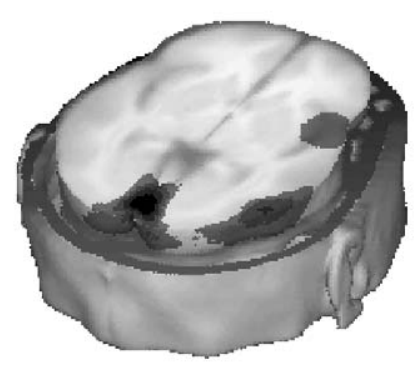

BMA
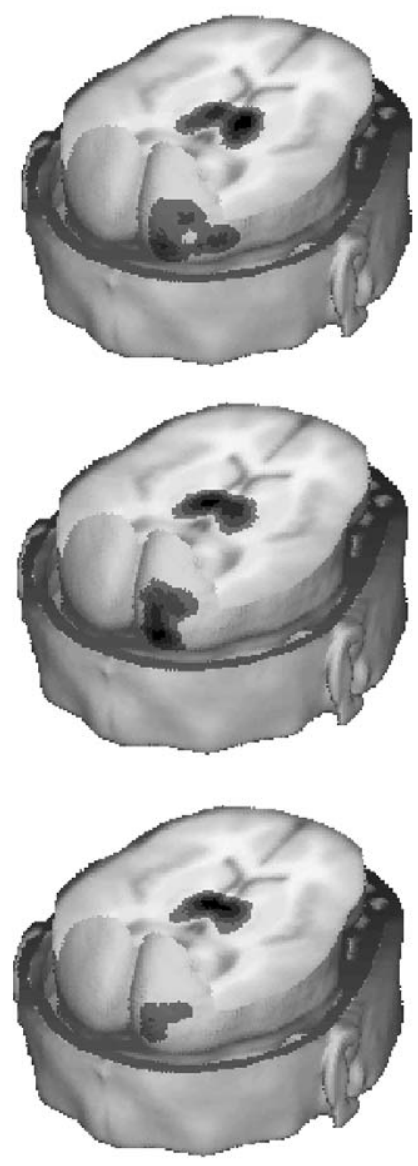

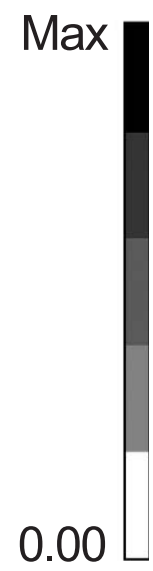

MEG-151
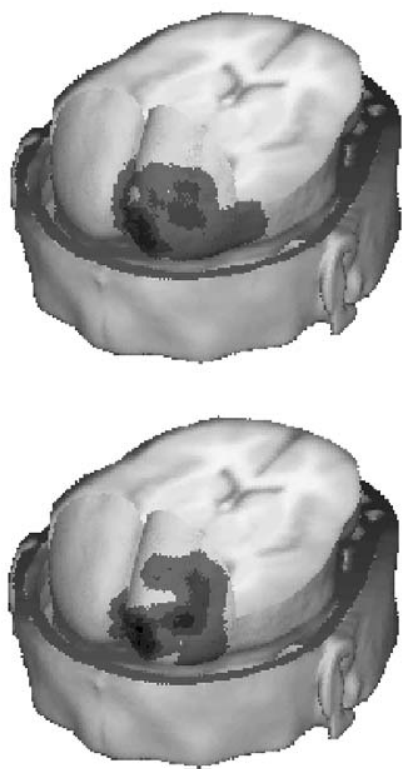

0.00

Fig. 8. 3D reconstructions of the absolute values of BMA and cLORETA solutions for the OPr + Th source case. The first column indicates the array of sensors used in each simulated dataset. The maximum of the scale is different for each case. For cLORETA (from top to down): max $=0.21,0.15$ and 0.05 ; for BMA (from top to down): $\max =0.41,0.42$ and 0.27 . 
The FWHM volume for this same dataset is shown in logarithmic scale in Fig. 5. In all cases, three different regions are clearly defined, depending on the FWHM values for each method. There are differences of two orders of magnitudes between the FWHM shown by BMA $\left(10^{-1}-10^{0} \%\right)$ and by LORETA $\left(10^{1}-10^{2} \%\right)$, while cLORETA shows values in an intermediate scale $\left(10^{\circ}-\right.$ $\left.10^{1} \%\right)$. Different trends with increasing eccentricity are also appreciated. That is, while BMA is relatively independent of the source eccentricity and of the number of sensors, with fluctuations in the order of tenths of units $\left(10^{-1} \%\right)$, LORETA undergoes changes from near $40 \%$ for deep sources to values below $10 \%$ for locations near the surface of the head (except for EEG-19/tangential case, in which small changes are observed). In the case of cLORETA, the FWHM reaches a maximum at medium eccentricities. For radial orientations this maximum is localized at $10 \%$ with amplitude of $5 \%$, while for tangential dipoles it moves to an eccentricity of $40 \%$, with slightly increased amplitude. Major effects related to the array of electrodes used are observed for eccentricities above $50 \%$, where both LORETA and cLORETA show reduced values of the FWHM when the number of sensors is increased.

Fig. 6 summarizes reconstruction results for MEG-151 measurements. As can be seen, the localization errors for BMA and
Table 1

BMA results for the two illustrative examples

\begin{tabular}{lllll}
\hline $\begin{array}{l}\text { Simulated } \\
\text { source }\end{array}$ & $\begin{array}{l}\text { Type of } \\
\text { sensors }\end{array}$ & $\begin{array}{l}\text { Number of } \\
\text { models in } C\end{array}$ & $\begin{array}{l}\text { Minimum and } \\
\text { maximum } \\
\text { probabilities in } C\end{array}$ & $\begin{array}{l}\text { Probability of } \\
\text { the true model }\end{array}$ \\
\hline Opr + Th & EEG-19 & 15 & $0.02-0.30$ & $0.11(3)$ \\
& EEG-120 & 2 & $0.49-0.51$ & $0.49(2)$ \\
Th & MEG-151 & 1 & $1.00-1.00$ & 1 \\
& EEG-19 & 3 & $0.37-0.30$ & $0.30(3)$ \\
& EEG-120 & 1 & $1.00-1.00$ & 1 \\
& MEG-151 & 1 & $1.00-1.00$ & 1 \\
\hline
\end{tabular}

Here $C$ denotes the Occam's Window as defined in Occam's window section, with $N=20\left(O_{\mathrm{L}}=0\right.$ and $\left.O_{\mathrm{R}} \approx 3\right)$. In the last column, the number in parenthesis indicates the position of the true model when all the models in $C$ are ranked by probabilities.

cLORETA show a similar behavior as in the EEG-120 case. LORETA on the contrary presents several differences. For large eccentricities (above 80\%), it shows small errors values (below $10 \%$ ) which are of the same order as BMA and cLORETA. For deeper sources, the localization error increases linearly with depth and reaches values around $50 \%$. Note that no dipole orientation

\section{cLORETA}

\section{EEG-19}

EEG-120
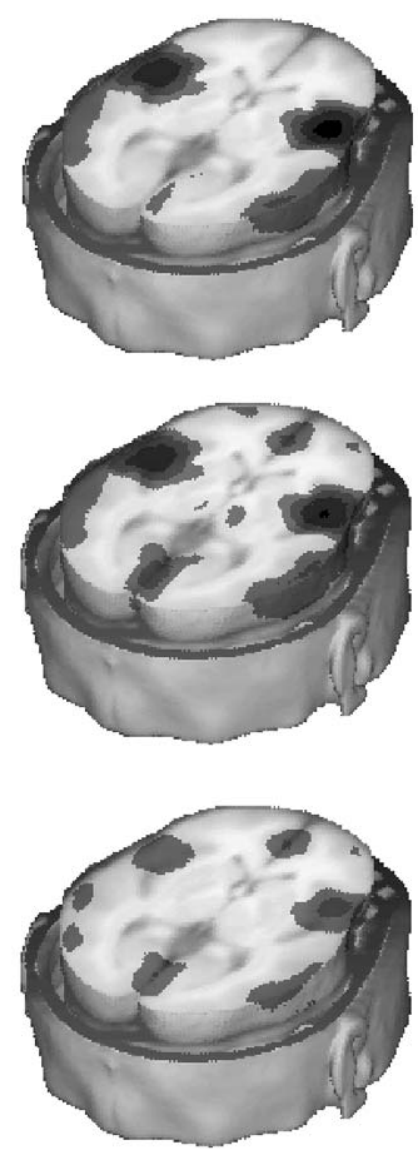

BMA
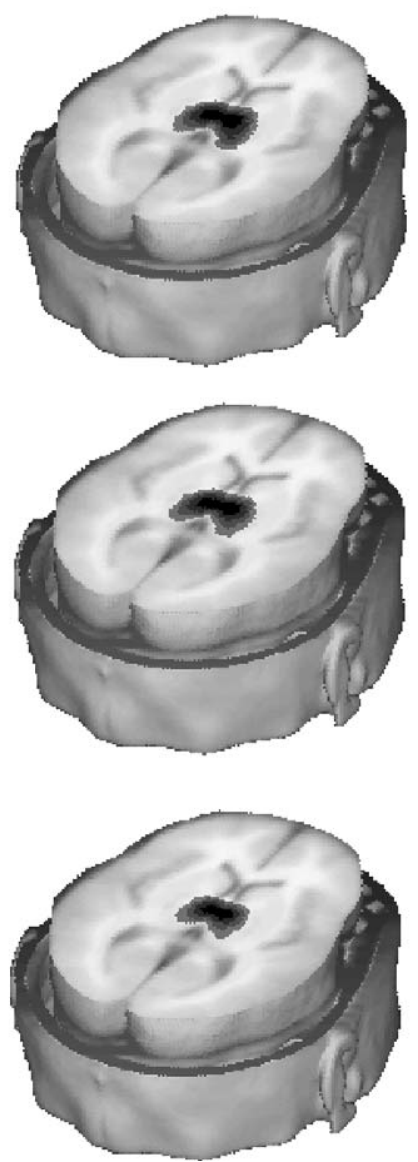

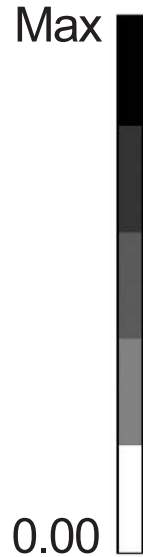

MEG-151

Fig. 9. 3D reconstructions of the absolute values of BMA and cLORETA solutions for the Th source case. The first column indicates the array of sensors used in each simulated dataset. The maximum of the scale is different for each case. For cLORETA (from top to down): $\max =0.06,0.01$ and $2.91 \times 10^{-3}$; for BMA (from top to down): $\max =0.36,0.37$ and 0.33 . 

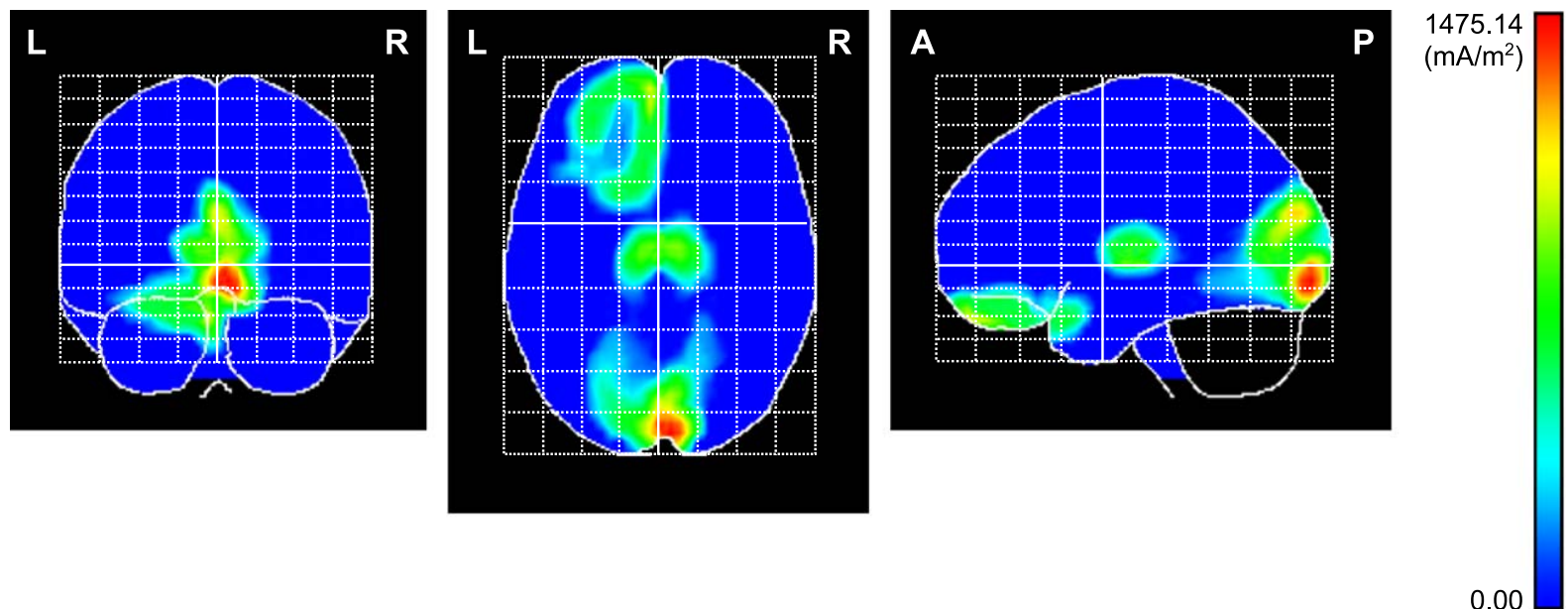

Fig. 10. Maximum intensity projection (onto coronal, axial and sagittal planes) views of the BMA solution for visual steady-state response to $19.5 \mathrm{~Hz}$ left eye stimulation. Three main sources are observed, with the maximum activity located in the calcarine fissure (occipital red spot). The frontal source covers the lateral and middle front-orbital gyrus and is associated with the electroretinogram produced by the activation of the photoreceptors in the retina. The third source is located in the thalamus.

effects are appreciated. Regarding the FWHM volume, the results shown by all approaches are very similar to those obtained for the EEG-120 case.

In summary, the BMA approach shows better tomographic properties than LORETA and cLORETA as regards localization error and spatial resolution (measured through the FWHM volume). Additionally, the values obtained for BMA are relatively independent on the number of sensors, the dipole orientation and even on the type of measurement.

\section{Two illustrative examples}

To show the performance of BMA regarding the problem with depth biasing and to demonstrate the limitations of linear solutions (exemplified through cLORETA) in this respect, two illustrative examples of source configurations are used. In the first case, two distributed sources are simulated at different eccentricity values at the same time. The outermost source is located at the occipital pole right, while the deeper one is placed at the thalamus (Opr $+\mathrm{Th})$.
The spatial distribution of the PCD is then simulated as two narrow Gaussian functions of the same amplitude; each of them peaked at the center of gravity of the corresponding region chosen for generating the EEG/MEG (Fig. 7A). The temporal dynamics already described in the case of dipole simulations is used for all the grid points within the two chosen regions. These same settings are then used for the second example, in which only the thalamic (Th) source was simulated (Fig. 7A). In both cases, the measurements are generated with $\mathrm{SNR}=10$.

The absolute values of the BMA and cLORETA solutions for the Opr + Th example and for the three arrays of sensors used are depicted in Fig. 8. In all cases, cLORETA is unable to recover the thalamic source, and blurred solutions plagued of ghost sources, which seems to be dominated by the cortical source, are shown instead. Note that the reconstructed sources become more concentrated and clearer as the number of sensors increases. On the contrary, more meaningful estimates of the PCDs are obtained when using BMA to analyze these data. As shown in the figure, the
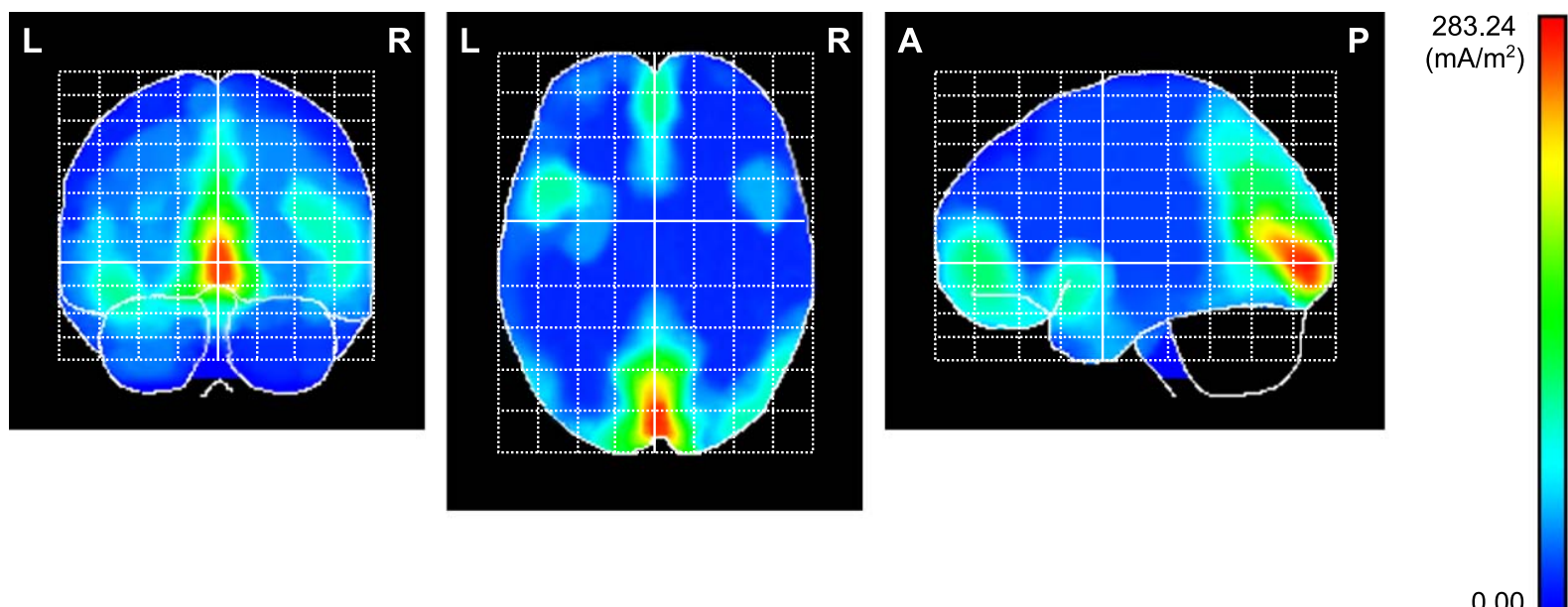

Fig. 11. Maximum intensity projection (onto coronal, axial and sagittal planes) views of the cLORETA solution for visual steady-state response to $19.5 \mathrm{~Hz}$ left eye stimulation. Maximum activity is located in the posterior pole of the brain (red spot). Four additional sources are visible in the axial plane, which are located at the insula and the inferior occipital and middle temporal gyri of both hemispheres. A sixth source in the anterior pole is also observed. 
spatial localizations of both cortical and subcortical sources are recovered with reasonable accuracy invariably. These results suggest that, unlike what have been believed by many authors, the EEG/MEG seems to contain enough information for estimating deep sources, even in cases where such generators might be hidden by cortical activations.

On the other hand, the reconstructed sources shown in Fig. 9 for the Th case demonstrate that depth biasing is an intrinsic problem of cLORETA, and not due to masking effects, because no cortical source is present in this set of simulations. Again in this example, the BMA approach gives significantly better estimates of the PCD.

An obvious question then arises: what makes cLORETA unable to fully exploit the information contained in the EEG? The answer to this question seems to be apparent when interpreting Minimum Norm-type methods within the framework of the Bayesian theory. The traditional estimation procedure followed by these methods is based on assuming that a given model (anatomical constraint) is true and then the parameters (the PCD inside the brain) and hyperparameters (regularization parameter) are estimated conditioned on that model. As we already know, this procedure is limited to the first two levels of the Bayesian inference paradigm and is able to give meaningful estimates of the PCD only in cases where the posterior probability of the model given the data is close to 1 (see Eq. (21)). In the present examples, the model that corresponds to cLORETA (the whole gray matter) was always rejected due to its low posterior probability and thus not included in the Occam's Window.

Some of the results of applying the BMA approach to these examples are summarized in Table 1 . Note that the number of models that belongs to the Occam's Window is reduced for increasing number of sensors. This is natural because more precise measurements imply more information available about the underlying phenomena, and then narrower and sharper model distributions are obtained. Consequently, as shown in the table, the probability and hence, the rank of the true model in the Occam's Window increases for dense arrays of sensors. Finally, note that in many cases, the model with the highest probability is not the true one. This fact supports the use of the BMA approach instead of using the model that maximizes the model probability distribution to carry out inference upon it. In the present simulations, this is not critical because the examples analyzed are too simple, but it becomes a determining factor when analyzing more complex data, as is the case with some real experimental conditions.

\section{Experimental data}

Now we shall investigate the results of applying the BMA approach to some experimental data. An important issue with this kind of testing is to find experimental scenarios where the results are as predictive as possible. To this end, we used four datasets coming from steady-state experiments that were designed to elicit responses from different brain areas related to the sensory system that was stimulated. Thus, responses from visual, somatosensory and auditory systems, to either left or right stimulation at different frequencies, were analyzed. In all cases, an array of 47 sensors (Fp1, Fp2, AF3, Afz, AF4, F7, F3, Fz, F4, F8, FC5, FC6, T7, C3, $\mathrm{Cz}, \mathrm{C} 4, \mathrm{~T} 8, \mathrm{CP} 5, \mathrm{CP} 1, \mathrm{CP} 2, \mathrm{CP} 6, \mathrm{P} 3, \mathrm{P} 7, \mathrm{Pz}, \mathrm{P} 4, \mathrm{P} 8, \mathrm{PO} 3, \mathrm{PO} 4$, $\mathrm{Pz}, \mathrm{O} 1, \mathrm{O} 2, \mathrm{Cb} 1, \mathrm{Cb} 2$ and $\mathrm{Iz}$ ) with $\mathrm{Cz}$ taken as reference electrode was used.

Before going further we wish to clarify that, because the main emphasis of this paper is on the estimation methods, we do not intend to make an exhaustive discussion of the results obtained in the experiments, which will be the subject of future publications.

\section{Visual steady-state response}

In this experiment, the stimulus consisted of flash stimulation to the left eye at a frequency of $19.5 \mathrm{~Hz}$. The maximum intensity projection onto the three orthogonal planes, corresponding to the absolute value of the posterior PCD obtained using BMA in this case, is depicted in Fig. 10. As seen in this figure, the maximum of the PCD is localized in the area of the primary visual cortex (PVC), which in humans lies almost exclusively on the medial surface of the posterior pole of the two cerebral hemispheres, on both sides of the calcarine fissure. This spatial distribution of the occipital generators agrees with the bilateral representation of the visual field in the PVC. As it is known, the right PVC receives input from the nasal hemiretina of the left eye, while the left PVC processes information from the temporal hemiretina. Consequently, visual field stimulation of the left eye should elicit activation of the PVC on both hemispheres.

The same reasoning can be applied to the almost symmetric activity seen in the thalamus. That is, the information flowing from the retina to the PVC is established through relay neurons lying on the lateral geniculate nucleus of the thalamus. It means that efferent fibers from the nasal hemiretina of the left eye make synapses in the contralateral side of the lateral geniculate nucleus, while left relay neurons are the target of fibers from the ipsilateral temporal hemiretina. Thus, the thalamus should reflect the same bilateral activity shown in the cortex. An additional frontal source in the lateral and middle front-orbital gyrus is also observed. This kind of source in the visual steady-state response has been commonly related to the electroretinogram (ERG) and is originated by activation of the photoreceptors and retinal middle layers. In summary, the tomographic map of the BMA solution shows an activation pattern that corresponds with the way in which the visual system processes information.

On the other hand, cLORETA, although showing a strong occipital peak very similar to the one obtained using BMA, also produces a cortical widespread activity with bilateral peaked amplitudes in the insula and the inferior occipital and middle temporal gyri (Fig. 11), which makes no sense from the physiological point of view. Note that, as in the simulations, this activity in the insula might suggest the existence of the additional thalamic source seen in the BMA solution. The ERG frontal source is also present in Fig. 11, but in this case it is located in the midline of the anterior pole, with activity that covers the cingulate region and the middle front-orbital gyrus of both hemispheres.

The results in Table 2 offer additional proof for the validity of the BMA approach over single model choice alternatives. Note that 29 models fall into Occam's Window, with a low value of the maximum posterior probability, which shows that there is no significant

Table 2

BMA results for the visual steady-state experiment

\begin{tabular}{lll}
\hline $\begin{array}{l}\text { Number of models in } \\
\text { the Occam's Window }\end{array}$ & $\begin{array}{l}\text { Maximum } \\
\text { probability }\end{array}$ & $p\left(M_{0} \mid \mathbf{v}\right)$ \\
\hline 29 & 0.09 & $1.24 \times 10^{-20}$ \\
\hline
\end{tabular}

Here the model $M_{0}$ contains the 71 compartments segmented, which corresponds to constrain the solution to the whole gray matter (cLORETA). The Occam's Window was defined by using $N=20\left(O_{\mathrm{L}}=0\right.$ and $\left.O_{\mathrm{R}} \approx 3\right)$ in Eq. (24) 

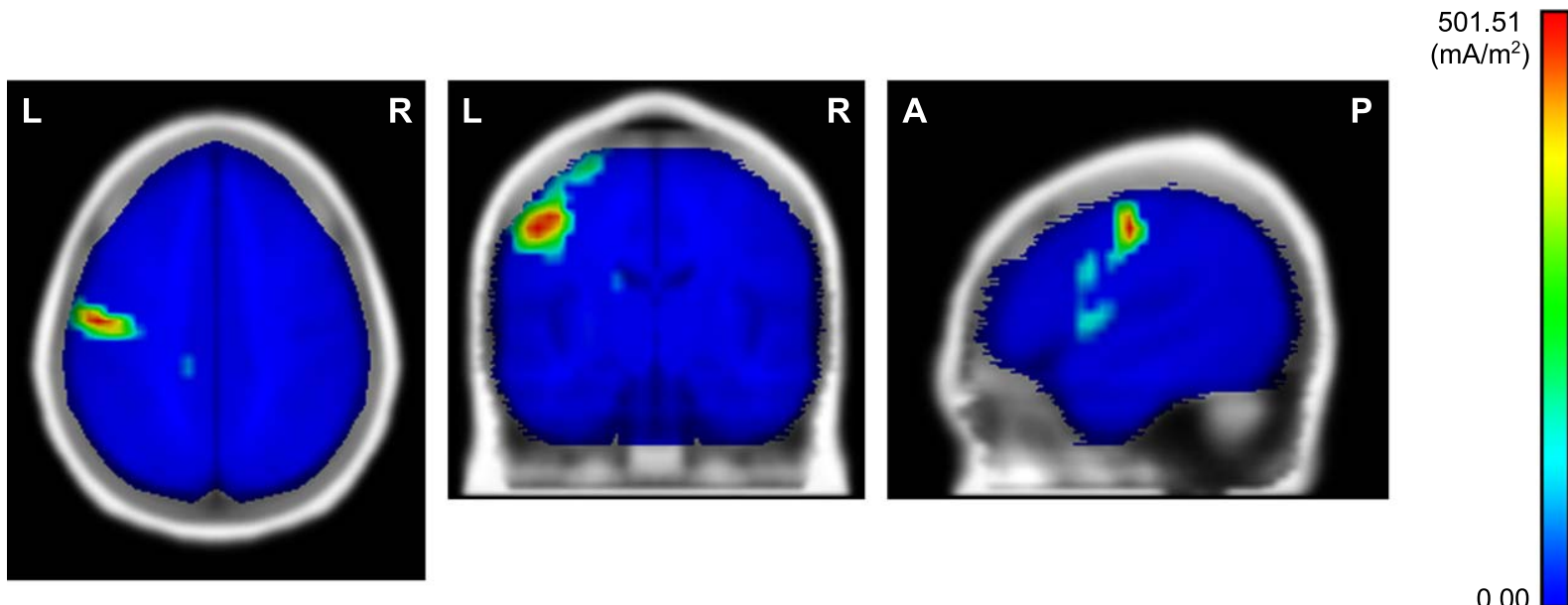

Fig. 12. Orthogonal views of the BMA solution for steady-state response to 23.4-Hz stimulation of the right thumb. The three planes intersect each other at the point of maximum activity, which is located in the postcentral gyrus left.

evidence in favor of any of these models. Thus, procedures based on making inference conditional on any single particular model in this case might lead to overconfident results and dangerous conclusions.
Finally, the low value of the posterior probability shown by the cLORETA model confirms the unreliability of this solution and the poor support that it receives from the data.
A
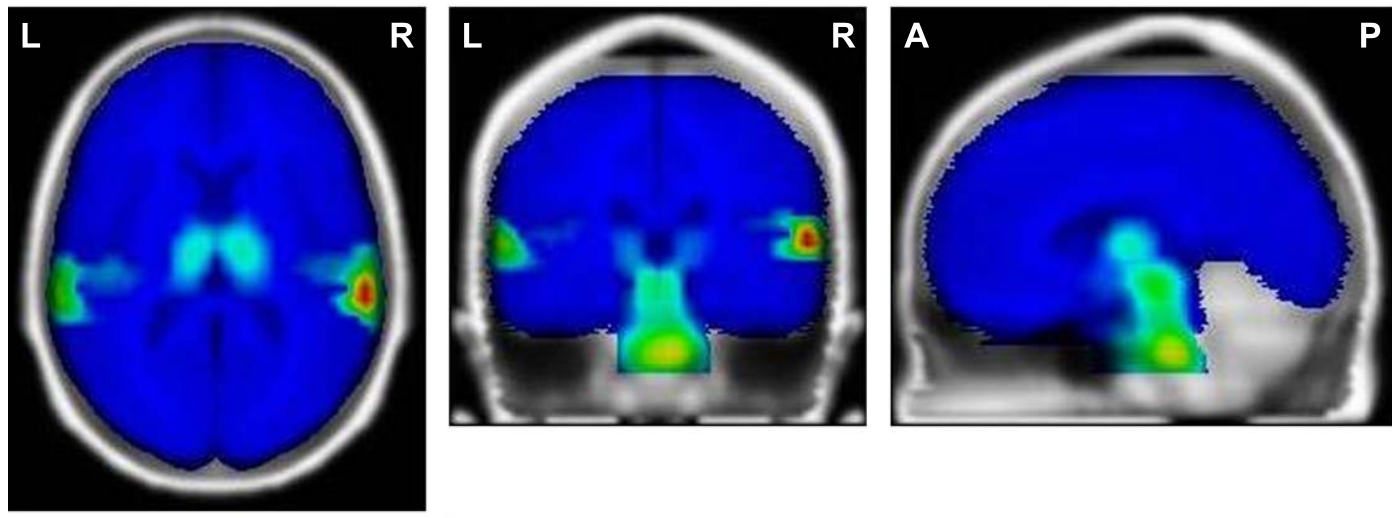

B

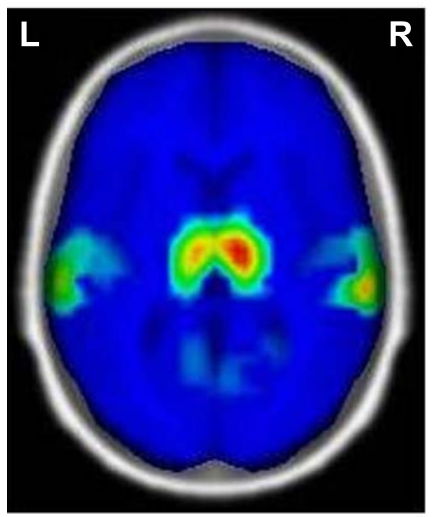

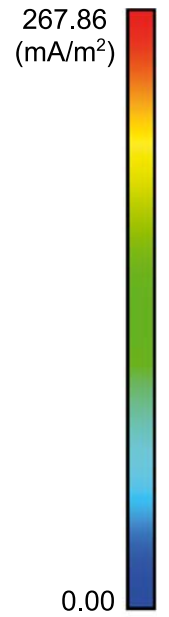

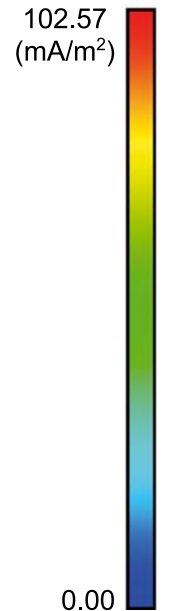

Fig. 13. Three orthogonal views of the BMA solution for auditory steady-state responses to left ear stimulation. (A) 12-Hz modulation frequency. (B) 90-Hz modulation frequency. In both, A and B, the axial cut was taken at the maximum of the activity (right auditory cortex and thalamus, respectively), while coronal and sagittal planes were used to visualize the brainstem source. 


\section{Somatosensory steady-state response}

In Fig. 12, the BMA solution for $23.4 \mathrm{~Hz}$ right thumb stimulation is shown. In this case, a contralateral activation clearly peaked in the area of the left primary somatosensory cortex (PSC) is observed. This spatial distribution of the generators is in correspondence with the contralateral organization of the somatosensory system. The ascending nerve fibers coming from peripheral receptors cross completely at the level of the medulla oblongata and make synapses in the contralateral cortex. According to this view, the left PSC contains information from the right side of the body because it receives no ipsilateral afferent input. Consequently, right somatosensory stimulation generates activation of the left PSC located in the postcentral gyrus of the left hemisphere, as is appreciated in the tomographic map of the BMA solution.

As in the visual steady-state data, the results of Table 3 validate the use of the BMA approach due to the great number of models that fall into Occam's Window and the low value of the maximum posterior probability of these models. In this case, again the model assumed by cLORETA was rejected due to the small value of its posterior probability.

\section{Auditory steady-state responses}

Intracerebral sources for auditory steady-state responses have not been extensively studied and are a matter of current debate among the neuroscientific community. Previous works using topographic mapping have reported polarity inversions of the response to $40 \mathrm{~Hz}$ brief tones over the midtemporal regions, suggesting that auditory cortex and some thalamocortical circuits might be involved in the generation of this kind of activity (Johnson et al., 1998). More recently, the use of dipole source analysis has revealed that both brainstem and cortical (temporal lobe) sources are active during steady-state responses when using modulation frequencies between 12 and $90 \mathrm{~Hz}$ (Picton et al., in press). In these studies, the intensity of the cortical activity was reported to decrease with increasing modulation frequency, with the brainstem being the dominant source at modulation rates greater than $50 \mathrm{~Hz}$. Thus, it seems to be a general agreement that auditory steady-state responses are generated in the auditory cortex and in subcortical structures, with the location of the maximum activity depending on the modulation frequency used in the stimulus.

The present data were obtained by stimulation of the left ear at modulation frequencies of 12 and $90 \mathrm{~Hz}$ and were recorded from 10 right-handed subjects with ages between 17 and 50 years old. A more detailed description of the experiment can be found in Herdman et al. (2002).

The absolute value of the BMA solution for the grand average over subjects at the two modulation rates is depicted in Fig. 13. Note that activations of brainstem, thalamus and

Table 3

BMA results for the somatosensory steady-state experiment

\begin{tabular}{lll}
\hline $\begin{array}{l}\text { Number of models in } \\
\text { the Occam's Window }\end{array}$ & $\begin{array}{l}\text { Maximum } \\
\text { probability }\end{array}$ & $p\left(M_{0} \mid \mathbf{v}\right)$ \\
\hline 15 & 0.12 & $6.33 \times 10^{-11}$ \\
\hline
\end{tabular}

As in Table 2, the model $M_{0}$ corresponds to cLORETA case, and the Occam's Window was defined by using $N=20\left(O_{\mathrm{L}}=0\right.$ and $\left.O_{\mathrm{R}} \approx 3\right)$ in Eq. (24). temporal lobe are obtained. For both frequencies, the cortical source exhibits bilateral activation of the superior temporal gyrus, with greater amplitude in the hemisphere contralateral to the stimulated ear. This same contralateral localization of the maximum activity was observed at the level of the thalamus. In contrast, the brainstem source showed an ipsilateral maximum at $90 \mathrm{~Hz}$, which changes into a more symmetric pattern for $12-\mathrm{Hz}$ modulation frequencies.

As expected from previous studies, the absolute maximum activity for $12 \mathrm{~Hz}$ was reached in the cortex, while deeper sources were dominant at $90 \mathrm{~Hz}$. However, there is one difference between the BMA solution presented here and the previous studies described above, which is related with the additional thalamic source that was found at both modulation rates. In this respect, the Bayesian framework shows results that seem to agree better with the anatomy of the auditory system because the three structures that were active are involved in the processing of auditory information. In other words, if both ends of the auditory pathway (brain stem and cortex) have been reported to take part in the generation of the steady-state response, it is intuitive to think that relay neurons located in the thalamus are also able to produce measurable electric activity over the scalp surface.

Finally, we want to note that the cLORETA model was rejected in these calculations due to its low posterior probabilities, which were of $7.54 \times 10^{-4}$ and $0.18 \times 10^{-2}$ for $12-$ and $90-\mathrm{Hz}$ modulation rates, respectively.

\section{Summary and discussion}

In this paper, a new formulation of the EEG/MEG Inverse Problem was described within the framework of the Bayesian inference theory, which provided for a high degree of flexibility and a suitable way for including prior information in the problem of finding unique generators of the EEG/MEG. This new formulation gave also a common ground of analysis for the wide range of inverse methods available. The main contribution of the present work, however, consisted of considering a third level of inference, now called Bayesian model averaging (BMA), that has been systematically omitted in previous works and where the Bayesian theory is in a class of its own. This level of inference allowed accounting for the uncertainty about assuming a given model as the truth and carrying out inference upon it, which is the usual practice for solving the EEG/MEG inverse problem. BMA then allowed us to compute posterior estimates of the PCD inside the brain unconditionally for any model considered.

This general formulation was used to address two of the main problems that affect linear inverse solutions: (a) the presence of ghost sources in the estimated solution and (b) the tendency to underestimate deep generators in favor of cortical ones. To this end, the Bayesian framework was applied to the case of considering different models, each differing in the anatomical constraints used to solve the EEG/MEG inverse problem. As a result, the final solution was calculated as a weighted average of the individual PCD for each particular model, where the weights were defined as the posterior probability of the corresponding model given the data. The BMA approach then favors brain regions that receive more support from the data and penalizes those which 
are less probable to contribute to the generation of the EEG/ MEG. The results of the current work demonstrate that this strategy seems to cope with the two critical problems described above.

This essential conclusion contradicts the widespread idea that deep subcortical structures are unable to generate a measurable voltage over the scalp surface. The reason for this belief rests on the fact that electric and magnetic fields are inversely related to the square of the distance, suggesting that the fields generated by deep sources decay fast enough to produce no detectable voltage at sensor locations. In contrast to this, the high posterior probability values obtained for models that included deep structures like the thalamus show that BMA strategy could offer a way to handle this problem. This might also suggests that somehow EEG/MEG contains the necessary information to estimate deep sources, which supports the claims of many authors in this field (Ioannides, 1994; Taylor et al., 1999). This paper is a step forward in that direction, although much work still needs to be done to give more conclusive assertions in this respect.

To characterize the new approach in terms of localization error and spatial resolution (FWHM volume), a simulation study was carried out, and the results were compared to LORETA and cLORETA solutions. It was demonstrated that BMA systematically improves the tomographic properties of these two previous approaches. Note that the frequency components used in the simulations belong to a narrow frequency band around $10 \mathrm{~Hz}$. Other frequency values were also used but not shown, given that very similar results were obtained.

Additionally, three types of actual data were analyzed, which covered a wide range of frequency values: visual $(19.5 \mathrm{~Hz})$, somatosensorial $(23.4 \mathrm{~Hz})$ and auditive $(12$ and $90 \mathrm{~Hz})$ steadystate responses. In all cases, meaningful results that seem to agree reasonably well with the neural substrate underlying those brain processes were obtained. For the visual experiment, a comparison with the solutions estimated by cLORETA was also carried out, demonstrating in this way some of the limitations of linear approaches in real experimental conditions.

The thrust of this paper is in the same direction as that of Schmidt et al. (1999). Both their paper and ours consider other properties of the posterior distribution than its mode, which is the usual case of maximum a posteriori Bayesian estimation. However, our formulation presented here differs from that of Schmidt in several ways. Regarding the activity model, Schmidt's choice consisted of semiparametric modeling of the $\mathrm{PCD}$, which was constrained to be regions on the cortical surface only, without regarding deeper sources. This parameterization defined the number, extension and location of the sources. In our case, modeling is nonparametric over a set of alternative models defined by the areas allowed to be active. Possible areas are defined from a previous parcelation of the cortex. There are also differences with respect to the manner in which the Bayesian formalism is exploited. In Schmidt's paper, estimation is carried out by sampling the posterior distribution of the parameters using MCMC and constructing histograms of each parameter marginalized with respect to the others. Attention is centered on presenting (a) the number of active sources and (b) the areas that appear with a given probability as activated. The use of evidence for each alternative model sampled is not explicitly addressed. Our procedure, on the other hand, is concerned with obtaining an explicit estimate for the activation strength of the sources by averaging alternative models, each of them weighted by its posterior probability. Undoubtedly these approaches may be combined. It is conceivable to do semiparametric modeling using a varying number of active regions with variable locations and extensions and then carry out model averaging. This possibility is currently under research.

We want to remark that the particular application of the BMA framework described here must be interpreted as a general way of introducing anatomical prior information in the solution of the EEG/MEG inverse problem by considering a higher level of inference within the Bayesian paradigm. In our case, the methodology is applied to linear distributed inverse solutions, but it can be equally applied to other kinds of methods. In Fuchs et al. (1999) for example, two implementations of a nonlinear approach based on using L1 norm instead of Frobenius' in Eq. (4) are proposed and compared with LORETA and Minimum Norm Least Square methods (MNLS). As reported in that paper, L1 norm outperforms LORETA with respect to the spatial resolution, measured through the FWHM volume. For a conjugate gradient implementation, the FWHM fluctuates between $1 \%$ and $9 \%$, while it is reduced to less than $1 \%$ for a sparse implementation. Nevertheless, the localization error for SNR below 10 (which is the case analyzed in the present paper) is in both cases greater than LORETA, and it shows a trend with the eccentricity in which cortical dipoles are much better localized than deep ones. The simulation results shown in this work demonstrate that the application of the BMA approach to linear methods improves these numbers. Smaller localization errors with relative independence of the eccentricity of the source are obtained and the spatial resolution is reduced to values comparable to those of the L1 norm sparse implementation. In this sense, we infer that the application of the BMA paradigm to nonlinear approaches (like the one proposed by Fuchs) could significantly improve its performance.

Finally, although the theory presented in this paper was described for the EEG/MEG inverse problem in the frequency domain, the time domain case can be easily considered by assuming real normal distributions for the likelihood and prior densities of the PCD, instead of complex ones. The main limitation of this simple model is that it does not include prior information about the temporal evolution of the activity, which would be a natural extension of the method. In general, the use of the three levels of the Bayesian inference framework opens a wide spectrum of possibilities that can be exploited not only for EEG/MEG and other techniques, but also for the analysis of joint recording data and the combination of information coming from different neuroimaging modalities.

\section{Acknowledgments}

We thank Dr. Alan Evans and his group from the McConnell Brain Imaging Center at the Montreal Neurological Institute for their important contribution to the present work by providing the Probabilistic Atlas used in the calculations. We also thank Dr. Terrence Picton from the Rotman Research Institute at Toronto University, who kindly offered the steadystate data analyzed in this paper and for his clear-minded comments on the results obtained. 


\section{Appendix A}

\section{A.1. Calculation of evidence for hyperparameters $\sigma$ and $\beta$}

With the choices of Eqs. (8) and (9) for the likelihood and the prior probability of $\boldsymbol{j}$, we obtain for the posterior probability

$$
\begin{aligned}
p\left(\boldsymbol{j} \mid \mathbf{v}, \sigma, \beta, M_{k}\right)= & \frac{1}{p\left(\mathbf{v} \mid \sigma, \beta, M_{k}\right)} \frac{1}{Z_{\mathbf{v}} Z_{\mathbf{j}}} \\
& \times \exp \left(-\left(\sigma\|\mathbf{v}-\mathbf{K} \boldsymbol{j}\|^{2}+\beta\|\mathbf{H} \boldsymbol{j}\|^{2}\right)\right)
\end{aligned}
$$

where $Z_{\mathbf{v}}=\left(\frac{\pi}{\sigma}\right)^{N_{\mathrm{s}}}, Z_{\mathbf{j}}=\left(\frac{\pi}{\beta}\right)^{3 N_{\mathrm{g}}}$. In Eq. (A1) we have also assumed that the complex multinormal density of a random vector $\boldsymbol{x}_{p \times 1}$ with mean $\boldsymbol{\mu}_{p \times 1}$ and covariance matrix $\boldsymbol{\Sigma}_{p \times p}$ is given by

$N_{p}^{\mathrm{c}}(\boldsymbol{\mu}, \Sigma)=\pi^{-p}|\Sigma|^{-1} \exp \left(-\left\|\boldsymbol{\Sigma}^{-1 / 2}(\mathbf{x}-\boldsymbol{\mu})\right\|^{2}\right)$

Adding and subtracting the term $\sigma^{2} \overline{\mathbf{v}}^{\mathrm{T}} \mathbf{K}\left(\sigma \mathbf{K}^{\mathrm{T}} \mathbf{K}+\beta \mathbf{H}^{\mathrm{T}} \mathbf{H}\right)^{-1} \mathbf{K}^{\mathrm{T}} \mathbf{v}$ to the exponent argument in Eq. (A1) and rearranging terms, we obtain

$$
\begin{aligned}
p\left(\boldsymbol{j} \mid \mathbf{v}, \sigma, \beta, M_{k}\right)= & \frac{1}{p\left(\mathbf{v} \mid \sigma, \beta, M_{k}\right) Z_{\mathbf{v}} Z_{j}} \exp \left(-\sigma\|\mathbf{v}\|^{2}\right. \\
& \left.+\left\|\mathbf{A}^{1 / 2} \hat{\boldsymbol{j}}_{k}\right\|^{2}-\left\|\mathbf{A}^{1 / 2}\left(\boldsymbol{j}-\hat{\boldsymbol{j}}_{k}\right)\right\|^{2}\right)
\end{aligned}
$$

Here $\mathbf{A}=\sigma \mathbf{K}^{\mathrm{T}} \mathbf{K}+\beta \mathbf{H}^{\mathrm{T}} \mathbf{H}$ and $\hat{\boldsymbol{j}}_{k}$ is given by Eq. (11). From Eq. (A2) we see that the posterior density for $\boldsymbol{j}$ is proportional to a complex multinormal distribution with mean $\mathrm{E}\left(\boldsymbol{j} \mid \mathbf{v}, \sigma, \beta, M_{k}\right)$ and covariance matrix $\operatorname{Var}\left(j \mid \mathbf{v}, \sigma, \beta, M_{k}\right)=\mathbf{A}^{-1}$. From the normalization condition, the posterior density $p\left(\boldsymbol{j} \mid \mathbf{v}, \sigma, \beta, M_{k}\right)$ in Eq. (A2) must integrate to 1 and then we obtain an expression for the evidence $p\left(\mathbf{v} \mid \sigma, \beta, M_{k}\right)$

$$
\begin{aligned}
p\left(\mathbf{v} \mid \sigma, \beta, H_{k}\right)= & \frac{\exp \left(-\sigma\|\mathbf{v}\|^{2}+\left\|\mathbf{A}^{1 / 2} \hat{\boldsymbol{j}}_{k}\right\|^{2}\right)}{Z_{\mathbf{v}} Z_{\mathbf{j}}} \\
& \times \int \exp \left(-\left\|\mathbf{A}^{1 / 2}\left(\boldsymbol{j}-\hat{\boldsymbol{j}}_{k}\right)\right\|^{2}\right) \mathrm{d} \boldsymbol{j}
\end{aligned}
$$

The integral here can be easily calculated because it is the normalizing constant of a complex multinormal distribution. Thus, the expression for the evidence takes the form

$$
\begin{aligned}
p\left(\mathbf{v} \mid \sigma, \beta, M_{k}\right)= & \left(\frac{\sigma}{\pi}\right)^{N_{\mathrm{s}}} \beta^{3 N_{\mathrm{g}}}|\mathbf{A}|^{-1} \\
& \times \exp \left(-\sigma\|\mathbf{v}\|^{2}+\left\|\mathbf{A}^{1 / 2} \hat{\boldsymbol{j}}_{k}\right\|^{2}\right)
\end{aligned}
$$

\section{A.2. Bayes' factors}

Let's assume that our data $\mathbf{v}$ comes from one of the two hypothesis $M_{0}$ and $M_{1}$, with a probability density $p\left(\mathbf{v} \mid M_{0}\right)$ and $p\left(\mathbf{v} \mid M_{1}\right)$. The inclusion of prior probabilities $p\left(M_{1}\right)$ and $p\left(M_{0}\right)=$ $1-p\left(M_{1}\right)$ then produces posterior probabilities $p\left(M_{1} \mid \mathbf{v}\right)$ and $p\left(M_{0} \mid \mathbf{v}\right)$
$=1-p\left(M_{1} \mid \mathbf{v}\right)$ through the consideration of the data. From the Bayes' Theorem, we obtain

$p\left(M_{k} \mid \mathbf{v}\right)=\frac{p\left(\mathbf{v} \mid M_{k}\right) p\left(M_{k}\right)}{p\left(\mathbf{v} \mid M_{0}\right) p\left(M_{0}\right)+p\left(\mathbf{v} \mid M_{1}\right) p\left(M_{1}\right)} \quad(k=1,2)$

Converting to the odds scale (odds = probability $/(1-$ probability $)$ ), we have

$\frac{p\left(M_{1} \mid \mathbf{v}\right)}{p\left(M_{0} \mid \mathbf{v}\right)}=\frac{p\left(\mathbf{v} \mid M_{1}\right) p\left(M_{1}\right)}{p\left(\mathbf{v} \mid M_{0}\right) p\left(M_{0}\right)}$

In this expression, the factor

$B_{10}=\frac{p\left(\mathbf{v} \mid M_{1}\right)}{p\left(\mathbf{v} \mid M_{0}\right)}$

which transforms the prior odds into posterior odds is called Bayes' factor.

\section{References}

Baillet, S., Garnero, L., 1997. A Bayeasian approach to introducing anatomo-functional priors in the EEG/MEG inverse problem. IEEE Trans. Biomed. Eng. 44, 374-385.

Bosch-Bayard, J., Valdés-Sosa, P., Virués-Alba, E., Aubert-Vázquez, E., John, R., Harmony, T., Riera-Díaz, J., Trujillo-Barreto, N., 2001. 3D statistical parametric mapping of variable resolution electromagnetic tomography (VARETA). Clin. Electroencephalogr. 32 (2), 47-66.

Brillinger, D.R., 1975. Time series: Data analysis and theory. (International Series in Decision Processes) Holt, Rinehart and Winston, Inc. Printed in the USA. ISBN 0-03-076975-2.

Clarke, C.J.S., 1991. Probabilistic modeling of continuous current sources. In: Rajala, H.-M., Katila, T. (Eds.), Biomagnetic Localization and 3D Modelling. Helsinki University of Technology, Department of Technical Physics, Helsinki, pp. 117-125.

Clarke, C.J.S., 1994. Error estimates in the biomagnetic inverse problem. Inverse Probl. 10, 77-86.

Collins, D.L., Neelin, P., Peters, R.M., Evans, A.C., 1994. Automatic 3D intersubject registration of MR volumetric in standardized Talairach space. J. Comput. Assist. Tomogr. 18, 192-205.

Dale, A., Sereno, M., 1993. Improved localization of cortical activity by combining EEG and MEG with MRI cortical surface reconstruction: a linear approach. J. Cogn., 162-176.

Dale, A.M., Liu, A.K., Fischl, B.R., Buckner, R.L., Belliveau, J.W., Lewine, J.D., Halgren, E., 2000. Dynamic statistical parametric mapping: combining fMRI and MEG for high-resolution imaging of cortical activity. Neuron 26 (1), 55-67.

De Munck, J.C., 1989. Random dipoles as a model for spontaneous EEG and MEG activity. In: Williamson, S.J., et al., (Eds.), Advances in Biomagnetism. Plenum, New York, pp. 595-598.

Draper, D., 1995. Assessment and propagation of model uncertainty (with Discussion). J. R. Stat. Soc., B 57, 45-97.

Evans, A.C., Collins, D.L., Mills, S.R., Brown, E.D., Kelly, R.L., Peters, T.M., 1993. 3D statistical neuroanatomical models from 305 MRI volumes. Proc. IEEE-Nuclear Science Symposium and Medical Imaging Conference, vol. 95. M.T.P Press, London, pp. 1813-1817.

Evans, A.C., Collins, D.L., Neelin, P., MacDonald, D., Kamber, M., Marrett, T.S., 1994. Three-dimensional correlative imaging: applications in human brain mapping. In: Thatcher, R., Hallet, M., Zeffiro, T., John, E.R., 
Huerta, M. (Eds.), Functional Neuroimaging: Technical Foundations. Academic Press, New York, pp. 145-161.

Everitt, B.S., Bullmore, E.T., 1999. Mixture model mapping of brain activation in functional magnetic resonance images. Hum. Brain Mapp. 7, $1-14$.

Friston, K.J., 2002. Bayesian estimation of classical systems: an application to fMRI. NeuroImage $16,513-530$.

Friston, K.J., Glaser, D.E., Henson, R.N.A., Kiebel, S., Phillips, C., Ashburner, J., 2002a. Classical and Bayesian inference in neuroimaging: applications. NeuroImage 16, 484-512.

Friston, K.J., Penny, W., Phillips, C., Kiebel, S., Hinton, G., Ashburner, J., 2002b. Classical and Bayesian inference in neuroimaging: theory. NeuroImage $16,465-483$.

Geweke, J., 1994. Bayesian comparison of econometric models. Econometrica 57, 1317-1340.

Fuchs, M., Wagner, M., Wischman, H.A., Dossel, O., 1995. Cortical current imaging by morphologically constrained reconstructions. In: Baumgartner, C., et al. (Eds.), Biomagnetism: Fundamental Research and Clinical Applications. Elsevier, IOS Press, Amsterdam, pp. $320-325$.

Fuchs, M., Wagner, M., Kohler, T., Wischman, H.A., 1999. Linear and nonlinear current density reconstructions. J. Clin. Neurophysiol. 16 (3), 267-295.

Gorodnitzky, I., George, J.S., Schlitt, H.A., Lewis, P.S., 1992. A weighted iterative algorithm for neuromagnetic imaging. Proc. IEEE Satellite Symp. Neurisci. Technology. Lyon, France, pp. 60-64.

Gorodnistky, I., George, J.S., Rao, B., 1995. Neuromagnetic imaging with FOCUSS: a recursive weighted minimum-norm algorithm. Electroencephalogr. Clin. Neurophysiol. 95, 231-251.

Green, P., 1995. Reversible jump Markov chain Monte Carlo computation and Bayesian model determination. Biometrika 82, $711-732$

Gull, S.F., 1989. Developments in maximum entropy data analysis. In: Skilling, J. (Ed.), Maximum Entropy and Bayesian Methods. Cambridge 1988 Kluwer, Dordrecht, pp. 53-71.

Hämäläinen, M., Ilmoniemi, R., 1984. Interpreting measured magnetic fields of the brain: estimates of current distributions. Tech. Rep. TKK-F-A620, Helsinki Univ., Helsinki, Finland.

Hämäläinen, M.S., Ilmoniemi, R.J., 1994. Interpreting magnetic fields of the brain: minimum norm estimates. Med. Biol. Eng. Comput. 32, $35-42$.

Hansen, P.C., 1992. Analysis of discrete illposed problems by means of the Lcurve. SIAM Rev. 34, 561-580.

Herdman, A.T., Lins, O., Van Roon, P., Stapells, D.R., Scherg, M., Picton, T.W., 2002. Intracerebral sources of human auditory steady-state responses. Brain Topogr. 15 (2), 69-86.

Hoeting, J.A., Madigan, D., Raftery, A.E., Volinsky, C.T., 1999. Bayesian model averaging: a tutorial. Stat. Sci. 14 (4), 382-417.

Ioannides, A.A., 1994. Estimates of brain activity using magnetic field tomography and large scale communication within the brain. In: Ho, M.W., Popp, F.A., Warnke, U. (Eds.), Bioelectrodynamics and Biocommunication. World Scientific, Singapore, pp. 319-353.

Ioannides, A.A., Bolton, J.P.R., Hasson, R., Clarke, C.J.S., 1989. Localized and distributed source solutions for the biomagnetic inverse problem II. In: Williamson, S.J., et al., (Eds.), Advances in Biomagnetism. Plenum, New York, pp. 591-605.

Johnson, B.W., Weinberg, H., Ribary, U., Cheyne, D.O., Ancill, R., 1998. Topographic distribution of the $40 \mathrm{~Hz}$ auditory evoked-related potential in normal and aged subjects. Brain Topogr. 1, 117-121.

Kass, R.E., Raftery, A.E., 1994. Bayes Factors. Technical Report no. 254. Department of Statistics, University of Washington.

Lütkenhöner, B., Grave de Peralta, R., 1997. The resolution-field concept. Electroencephalogr. Clin. Neurophysiol., Electroencephalogr. Clin. Neurophysiol. 102, 326-334.

MacKay, D.J.C., 1992. Bayesian interpolation. Neural Comput. 4 (3), $415-447$.

Madigan, D., Raftery, A., 1994. Model selection and accounting for model uncertainty in graphical models using Occam's window. J. Am. Stat. Assoc. 89, 1535-1546.

Madigan, D., York, J., 1992. Bayesian graphical models for discrete data. Technical Report no. 259. Department of Statistics, University of Washington.

Matsuura, K., Okabe, Y., 1995. Selective minimum-norm solution of the biomagnetic inverse problem. Proc. IEEE Trans. Biomed. Eng. 42, $608-615$.

Matsuura, K., Okabe, Y., 1996. Lp-normalized Minimum L1-norm solution of the biomagnetic inverse problem. Proc. IEEE Eng. Med. Biol. Soc.. Nijmegen, The Netherlands, p. 145.

Matsuura, K., Okabe, Y., 1997. A robust reconstruction of sparse biomagnetic sources. IEEE Trans. Biomed. Eng. 44, 720-726.

Mazziotta, J.C., Toga, A., Evans, A.C., Fox, P., Lancaster, J., 1995. A probabilistic atlas of the human brain: theory and rationale for its development. NeuroImage 2, 89-101.

Nunez, P., 1981. Electric Fields of the Brain: The Neurophysics of EEG. Oxford Univ. Press, New York.

Pascual-Marqui, R.D., 1995. Reply to comments by Hämäläinen, Ilmoniemi and Nunez. In: Skrandies, W. (Ed.), Source Localization: Continuing Discussion of the Inverse Problem. ISBET Newsletter, vol. 6, pp. 16-28 (ISSN 0947-5133).

Pascual-Marqui, R.D., 1999. Review of methods for solving the EEG inverse problem. Int. J. Bioelectromagn. 1 (1), 75-86.

Pascual-Marqui, R.D., Michel, C.M., Lehman, D., 1994. Low resolution electromagnetic tomography: a new method for localizing electrical activity of the brain. Int. J. Psychophysiol. 18, 49-65.

Picton, T.W., Alain, C., Woods, D.L., John, M.S., Scherg, M., Valdés-Sosa, P., Bosch-Bayard, J., Trujillo, N.J., 1999. Intracerebral sources of human auditory-evoked potentials. Audiol. Neuro-otol. 4, 64-79.

Picton, T.W., John, M.S., Dimitrijevic, A., Purcell, D., 2003. Human auditory steady-state responses. Rev. Pap. Int. J. Audiol. 42, 177-219.

Plonsey, R., 1963. Reciprocity applied to volume conductors and the ECG. IEEE Trans. Bio-Med. Electron. 10, 9-12.

Raftery, A.E., 1993. Bayesian model selection in structural equation models. In: Bollen, K.A., Long, J.S. (Eds.), Testing Structural Equation Models. Sage, Beverly Hills, pp. 163-180.

Raftery, A.E., Madigan, D., 1997. Bayesian model averaging for linear regression models. J. Am. Stat. Assoc. 92, 179-191.

Raftery, A.E., Madigan, D., Hoeting, J.A., 1993. Model selection and accounting for model uncertainty in linear regression models. Technical Report no 262, Department of Statistics, University of Washington.

Riera, J.J., Fuentes, M.E., Valdés, P.A., Oharriz, Y., 1997a. Theoretical basis of the EEG spline inverse solutions for a spherical head model. Biomed. Tech. 42 (1), 219-222.

Riera, J., Valdés, P., Fuentes, M.E., Ohárris, Y., 1997b. Explicit Backus and Gilbert EEG inverse solution for a spherical head model. Biomed. Tech. 42 (1), 216-218.

Rush, S., Driscoll, D.A., 1969. EEG electrode sensitivity—An application of reciprocity. IEEE Trans. Biomed. Eng. 16 (1), 15-22.

Scherg, M., von Cramon, D., 1986. Evoked dipole source potentials of the human auditory cortex. Electroencephalogr. Clin. Neurophysiol. 65, $344-360$.

Schmidt, D.M., George, J.S., Wood, C.C., 1999. Bayesian inference applied to the biomagnetic inverse problem. Hum. Brain Mapp. 7, 195-212.

Scholz, B., Schwierz, G., 1994. Probability-based current dipole localization from biomagnetic fields. IEEE Trans. Biomed. Eng 41, $735-742$.

Smith, A.F.M., 1991. Contribution to the discussion of "Posterior Bayes factors". J. R. Stat. Soc., Ser. B 53, 132-133.

Srebro, R., 1996. An iterative approach to the solution of the inverse problem. Electroencephalogr. Clin. Neurophysiol. 98, 349-362.

Tarantola, A., 1987. Inverse Problem Theory. Elsevier, The Netherlands (ISBN 0-444-42765-1).

Taylor, J.G., Ioannides, A.A., Müller-Gärtner, H.W., 1999. Mathematical analysis of lead field expansions. IEEE Trans. Med. Imag. 18, $151-163$. 
Tikhonov, A.N., Arsenin, V.Y., 1977. Solutions of Ill-Posed Problems. W.H. Winston, Washington, DC.

Trujillo-Barreto, N.J., Martínez-Montes, E., Melie-Gracía, L., ValdésSosa, E., 2001. A symmetrical Bayesian model for fMRI and EEG/MEG neuroimage fusion. Int. J. Bioelectromagn. 3 (1).

Valdes-Sosa, P., Marti, F., Garcia, F., Casanova, R., 2000. Variable resolution electric-magnetic tomographyBiomag 96': Proceedings of the
Tenth International Conference on Biomagnetism, vol. II. Springer-Verlag, New York, pp. 373-376.

Vidakovic, B., 1998. Nonlinear wavelet shrinkage with Bayes rules and Bayes factors. J. Am. Stat. Assoc. 93, 173-179.

Wang, J.Z., Williamson, S.J., Kaufman, L., 1992. Magnetic source images determined by a leadfield analysis - The unique minimum-norm leastsquares estimation. IEEE Trans. Biomed. Eng. 39, 231-251. 